\title{
Astroplastic: A start-to-finish process for polyhydroxybutyrate production from solid human waste using genetically engineered bacteria to address the challenges for future manned Mars missions
}

\section{Author List}

Xingyu Chen (2), Syeda Ibrahim (2), Alina Kunitskaya (3), Kaitlin Schaaf (1), Zi Fei Wang (2), Preetha Gopalakrishan (2), Maliyat Noor (3), Harry Wilton-Clark (2), Jacob Grainger (2), Alexandra Ivanova (3), Patricia Lim (1), Michaela Olsakova (4), Lalit Bharadwaj (2), Bilal Sher (3), David Feehan (2), Rachelle Varga (1), Mayi Arcellana-Panlilio (2) *

1. Department of Biology, University of Calgary

2. Cumming School of Medicine, University of Calgary

3. Schulich School of Engineering, University of Calgary

4. Department of Computer Sciences, University of Calgary

*Corresponding author: Mayi Arcellana-Panlilio (myarcell@ucalgary.ca)

\section{Author Contributions}

Conceptualization: KS XC AK PG MAN HWC SI ZW AI MO JG PL LB BS RCV MAP

Methodology: AK KS XC SI MAN HWC JG AI PG ZW LB PL MO BS

Software: MO

Formal Analysis: AK MAN AI

Investigation: KS XC PG SI AK HWC MAN ZW LB JG PL MO RCV DF BS

Resources: MAP RCV AK DF

Writing Original Draft Preparation: XC SI AK ZW PG KS MAN HWC JG LB

Writing - Review \& Editing: XC AK SI ZW MAP LB HWC

Visualization: TL MO XC AK HWC

Supervision: MAP RCV DF

Project Administration: MAP RCV DF AK XC SI KS

Funding Acquisition: MAP RCV AK KS XC PG SI MO PL ZW LB MAN HWC BS JG 


\section{Abstract}

Space exploration has long been a source of inspiration, challenging scientists and engineers to find innovative solutions to various problems. One of the current focuses in space exploration is to send humans to Mars. However, the challenge of transporting materials to Mars and the need for waste management processes are two major obstacles for these long-duration missions.

To address these two challenges a process called Astroplastic was developed that produces polyhydroxybutyrate (PHB) from solid human waste, which can be used to 3D print useful items for astronauts. PHB granules are naturally produced by bacteria such as Ralstonia eutropha and Pseudomonas aeruginosa for carbon and energy storage. The phaJ, phaC, and phaCBA genes were cloned from these native PHB-producing bacteria into Escherichia coli. These genes code for enzymes that aid in PHB production by converting products of glycolysis and $\beta$-oxidation pathways, such as acetyl-CoA and enoyl-CoA, into PHB. To ensure a continuous PHB production system and to eliminate the need for cell lysis to extract PHB, recombinant $E$. coli was engineered to use the genes in its natural type I secretion system to secrete PHB. The C-terminal of the HlyA secretion tag was fused to phasin (PhaP), a protein originally from $R$. eutropha. Phasin-HlyA electrostatically binds PHB granules and transports them outside of the cell.

In addition to genetically engineering bacteria, a concept for start-to-finish PHB production process was designed. Integrating expert feedback and experimental results, conditions for each step of the process including the collection and storage of waste, volatile fatty acid (VFA) fermentation, VFA extraction, PHB fermentation, and PHB extraction were optimized. The optimized system will provide a sustainable and continuous PHB production system, which will address the problems of transportation costs and waste management for future space missions.

\section{Financial Disclosure}

Mindfuel Science Alberta Foundation

Genome Alberta

GenScript

Polyferm Canada

GeekStarter Alberta

Integrated DNA Technologies

University of Calgary

University of Calgary Cumming School of Medicine

University of Calgary Bachelor of Sciences

University of Calgary Schulich School of Engineering

University of Calgary O'Brien Centre for the Bachelor of Health Sciences 
This work was assessed during the iGEM/PLOS Realtime Peer Review Jamboree on $23^{\text {rd }}$ February 2018 and has been revised in response to the reviewers' comments. A transcript of these comments is available with the original article as a supplementary file

City of Calgary

Alberta Innovates

The funders had no role in study design, data collection and analysis, decision to publish, or preparation of the manuscript.

\section{Competing Interests}

The authors have declared that no competing interests exist.

\section{Ethics Statement}

$\mathrm{N} / \mathrm{A}$

\section{Data Availability}

All data are freely available without restriction.

\section{Introduction}

Governments and private enterprises alike are gearing up for travel across our Solar System. Plans to colonize nearby planets are underway, with Elon Musk spearheading the initiative to put a human colony on Mars by 2030 . Furthermore, NASA is planning a manned exploratory mission to Mars as soon as the 2030s. Several other space agencies have similar plans and timelines for their own respective Mars explorations. This exciting time in our history nonetheless comes with the challenges of long-term space travel.

Two current ecological and economic challenges for space travel include the sustainable management of waste produced in space and the high cost of shipping materials to space. Recycling of waste on Mars will be paramount because manned missions will need to recover as much water and oxygen as possible to sustain life. Human waste must also be treated to minimize health risks for the crew of a Mars mission. All of this must be accomplished while preserving the natural Martian environment. Transportation of goods is also a major economic barrier for longduration missions to Mars because the current cost of shipping materials up to low Earth orbit is $\$ 22,000$ USD per kilogram (\$10,000 USD per pound) [1]. This expense will constrain early Mars mission crews in the supplies that they can bring or ship from Earth to Mars and may not allow astronauts to account for every tool they may require during their mission. One way to mitigate this challenge is to develop a system to produce necessary items in space as needs arise from solid organic waste, an abundant resource from space travel.

This project worked on a unique solution to both challenges of future Mars missions; upcycle human waste by using it as a feedstock for $E$. coli engineered to produce bioplastic, which can then be 3D printed into useful tools on-site.

Poly(3-hydroxybutyrate) (PHB), a bioplastic, is produced in nature by many bacterial species. Literature has shown that PHB can be produced using a variety of feedstocks, including glucose and volatile fatty acids (VFAs) [2]. Since human waste contains both glucose and VFAs, it is a potentially useful feedstock for PHB production. The overarching goal for the synthesis component of the project was to produce PHB by utilizing the nutrients present in 
This work was assessed during the iGEM/PLOS Realtime Peer Review Jamboree on $23^{\text {rd }}$ February 2018 and has been revised in response to the reviewers' comments. A transcript of these comments is available with the original article as a supplementary file

human waste. To accomplish the efficient conversion of organic feedstocks into PHB, Escherichia coli were genetically engineered to produce PHB by manipulating the endogenous beta-oxidation and glycolysis pathway.

The choice of the genes and metabolic pathway was informed by the types of organic compounds available in human fecal waste that can serve as substrates for PHB synthesis. Analysis of solid human waste showed that human fecal waste contains VFAs (short-chain fatty acids such as acetic acid, propionic acid, and butyric acid), long chain fatty acids, and glucose which can be used as substrates for PHB production [3]. Therefore, the synthesis system must make use of a wide range of carbon sources and transform them into the desired product, PHB.

Many pathways and genes were manipulated in this study to use the organic substrates in human fecal waste to synthesize PHB $[4,5,6,7]$. To utilize the fatty acids found in human fecal waste, the fatty acid beta-oxidation pathway within $E$. coli was manipulated. A construct was designed to convert short- and medium-chain-length VFAs to PHB. This construct contained the phaJ4 gene from Pseudomonas putida, encoding for enoyl-CoA hydratase that converts the enoyl-CoA into (R)-hydroxybutyrate and the phaC1 gene, a gene from Pseudomonas aeruginosa encoding the PHA synthase, which converts the (R)-hydroxyacyl-CoA into polyhydroxybutyrate (PHB) [8]. This production pathway not only uses VFAs but can also use undigested long-chain fatty acids in human fecal waste, thus maximizing the substrates available for PHB synthesis.

Another production system construct contained the phaCBA genes, which uses the products of glycolysis for PHB production. The phaCBA pathway was adopted from the naturally existing phaCAB operon found in $R$. eutropha $\mathrm{H} 16$ which is involved in the biosynthesis of PHB [4]. Transcription of the phaCAB operon leads to expression of the following enzymes in the order: pha synthase (phaC), 3-ketothiolase (phaA), and acetoacetyl-CoA reductase (phaB). 3-ketothiolase converts acetyl-coA to acetoacetyl-CoA. These enzymes use acetyl-coA, which results from degradation of carbohydrates and VFAs, as a primary substrate to produce PHB [4]. The acetoacetyl-CoA reductase leads to conversion of acetoacetyl-CoA to (R)-3-hydroxybutyryl-CoA. Finally, pha synthase converts (R)-3hydroxybutyryl-CoA to PHB [4]. Hiroe et al. showed that the content of PHB is dependent on the expression of phaB [4]. Therefore, this construct contained the rearrangement from native phaCAB operon to phaCBA to promote higher expression of phaB for increased PHB produced.

Codon optimization helps to increase the translational efficiency of gene by modification of DNA sequence of one species into a sequence of codons most recognized another species. As a result, protein expression is maximized in the respective organism. Therefore, the constructs described above were codon optimized to increase their translational efficiency within E. coli.

To produce PHB, the synthetic feces (see methods) was prepared as a feedstock for the bacterial cultures. PHB is produced and stored as intracellular granules that range in size from 60-80 nm within the bacteria [9]. This creates a challenging problem: efficient extraction of the desired PHB from inside the cell. After the evaluation of the advantages and disadvantages of different extraction methods in terms of our space application, the Type I hemolysin secretion pathway, which is endogenous to E. coli, was selected as a method to extract PHB [9] due to its advantages over traditional extraction methods such as chemical lysis and heat-induced lysis.

Chemical lysis traditionally uses solvent extraction employing chemicals such as chloroform and sodium hypochlorite [10]. Although this method produces efficient yields and is relatively simple, it requires chemicals which need to be transported to and from Mars. This introduces a high cost for the project. In addition, this method requires the manual input of chemicals, which would demand time from astronaut's schedule. The chemical lysis 
This work was assessed during the iGEM/PLOS Realtime Peer Review Jamboree on $23^{\text {rd }}$ February 2018 and has been revised in response to the reviewers' comments. A transcript of these comments is available with the original article as a supplementary file

would kill the cells and eliminate the continuous production of PHB. In addition, the cellular debris will make the separation of the PHB more difficult. Heat-induced lysis was another method for PHB extraction which depends on the T4 lysis genes (holins and endolysins) under the control of the $\mathrm{pR}$ Lambda promoter with thermosensitive repressor proteins cl587. When heated, lysis genes become activated [11]. This system is advantageous because it does not require chemicals for lysis. However, the energy consumption from the heating of fermenters is an issue because energy is another limited resource in space. In addition, heat-induced lysis also kills the bacterial cells, not compatible with the goals of continuous and sustainable production and extraction system. Given the disadvantages associated with these conventional PHB extraction methods, the BBa_K2018024 from the SDU-Denmark 2016 was as the basis for the design of a Type I hemolysin secretion mechanism.

PHB is secreted through the hemolysin pathway that is endogenous to E. coli. Naturally, the hemolysin toxin (HlyA) is secreted from $E$. coli as a defence mechanism. This is a single-step process that involves three additional proteins: HlyB, HlyD, and TolC. HlyB is an active transport protein that uses ATP in the cytoplasmic membrane, $\mathrm{HlyD}$ is a membrane fusion protein that spans the periplasm and connects the inner and outer membrane proteins, and TolC rests in the outer membrane. When HlyB recognizes the C-terminus of HlyA, it stimulates formation of the secretion channel and the toxin is secreted [12].

To use this system in our biologically engineered cells, the C-terminus of $\mathrm{HlyA}$, which is endogenous to E. coli, was added to the end of a phasin molecule. Phasin, coded for by phaP, is a small structural protein found in bacteria that naturally produce PHB. It electrostatically binds to intracellular PHB, and therefore the PHB granules with bound phasin-HlyA tag are secreted as one by type 1 secretion machinery. Granules secreted through this mechanism range from 20-60 $\mathrm{nm}$ in size [9].

Part BBa_K2018024 from the SDU-Denmark 2016 team was used for the design of a Biobrick for PHB secretion. This part contains a coding region for phasin (phaP), originally from $R$. eutropha, as well as a coding region for the HlyA secretion tag $(h l y A)$, originally from $E$. coli. The entire sequence for $E$. coli was sequence-optimized to improve protein expression in the chassis, E. coli BL21(DE3), and restriction sites were removed to make it compatible with all iGEM RFC assembly standards. An IPTG-inducible T7 bacteriophage promoter was chosen to upregulate the production of Phasin-HlyA because increased production of Phasin has been shown to decrease PHB granule size, which could increase the efficiency of PHB translocation across the cellular membrane [13]. In addition, genes under the control of an inducible promoter have much higher rate of transcription than those that are constitutive. The $E$. coli BL21(DE3) genome contains a coding region for T7 RNA polymerase under the control of an IPTG-inducible promoter, and it was therefore chosen as an ideal chassis. Furthermore, a FLAG tag (amino acid sequence DYKDDDDK) was added to the $\mathrm{N}$-terminus of the Phasin-HlyA fusion protein for easier isolation of our protein during protein expression analyses.

Process design: In the first step of the process, astronaut's feces are collected into a storage tank using a vacuum toilet. Feces are then transferred into another tank and left to ferment for three days with natural gut flora to increase the concentration of volatile fatty acids (VFAs) that are later consumed by engineered E. coli to produce PHB. Next, the liquid containing VFAs is separated from solid particles using centrifugation and filtration. The resulting liquid containing VFAs is then passed to another storage tank. From there, VFAs are added to a fermenter inoculated with the engineered PHB-producing and PHB-secreting E. coli. Lastly, the resulting PHB is extracted from the liquid harvest stream. The PHB can then be used in a Selective Laser Sintering (SLS) 3D printer without the need for additional processing [14]. 
This work was assessed during the iGEM/PLOS Realtime Peer Review Jamboree on $23^{\text {rd }}$ February 2018 and has been revised in response to the reviewers' comments. A transcript of these comments is available with the original article as a supplementary file

During the development of the project, the team consulted experts to determine the most appropriate project application. When the feasibility of this project was evaluated, there was four possibilities of where to integrate PHB production: wastewater treatment plants, small-scale wastewater treatment plants in developing countries, landfill leachate treatments, and on Mars colonies with human solid waste as feedstock. The former three project applications were deemed unfeasible due to high up-front costs. After careful consideration, the team chose Mars colonies as the final project application.

From conversations with experts, such as Dr. Robert Thirsk and Col. Chris Hadfield (two Canadian astronauts), the team was alerted to considerations for designing systems for space. The team thus considered In-Flight Maintenance Requirements, where any machine that is brought to and used in space must be one that can be easily repaired by crew members. This aspect in design was taken into consideration by Process Development in designing the key components of the PHB-producing system. The team also made sure that the system was compliant with current policies and regulations, such as the treaties released by the United Nations Committee on the Peaceful Uses of Outer Space (COPUOS).

Based on COPUOS recommendations, the team emphasized containment of foreign biological systems in their project design. Dr. Nicole Buckley (a microbiologist at the Canadian Space Agency (CSA)) provided the team with possible safety concerns with the PHB-producing system, such as viral shedding from feces that can increase disease prevalence on board the spacecraft. To address this issue, the team is currently considering the implementation of UV treatment to assist in sterilizing the human waste after it has been fermented with natural gut flora. Dr. Buckley also indicated the possibility of having abnormal or decreased growth rates of bacteria in microgravity. To address this, it was decided that the PHB-producing system would be implemented under the gravitational pull of Mars in a colony. Furthermore, Dr. Buckley also expressed the difficulty of bring cell maintenance materials to space, which pushed the team towards the less material-intensive PHB secretion system, as opposed to a lysis system.

Dr. Matthew Bamsey (the Chief Systems Engineer at the German Aerospace Center) was also consulted; he provided NASA documents on Equivalent System Mass, which provides an universal method of calculating the required energy and efficiency of items brought to space, and mentioned that the team should consider the NASA Life Support Baseline Values and Assumptions, which is a document containing a set of values to be used in the Equivalent System Mass Analysis. Current applications of solid waste in space were also accounted for on the recommendation of Dr. Pascal Lee (the Principal Investigator of the Haughton-Mars Project at the NASA Ames Research Center).

Made in Space, Inc. is the company that developed and implemented the installation of a 3D printer currently in use on the ISS. Dr. Derek Thomas is the Senior Materials Scientist at this company and offered the team valuable advice for safety and encouraged the team to explore the flammability, off-gassing, and other physical characteristics of PHB on Mars. Secondary processing may also be a method to obtain desired properties not found in the unrefined PHB. Moreover, although Dr. Thomas did not foresee any major concerns with the use of powder PHB in a SLS 3D printer, it may be difficult to control the powder in microgravity. This is another reason why the team chose to apply the system on a Mars colony, where gravitational pull is present. The feasibility of using PHB as 3D printing feedstock was also supported by Dr. Bruce Ramsay, the founder and CTO of Polyferm, a commercial PHB production company. 


\section{Materials and Methods}

\section{Genetic Constructs}

\section{Codon Optimization}

Genes of interest were codon optimized for use in E. coli. This was done by breaking the DNA sequence of nucleotides into codons, which were replaced with codons of higher frequency in E.coli that translates to the same amino acids. The genes were optimized using the codon usage table for E. coli [15].

\section{Competent E. coli BL21 (DE3) and DH5 Preparation}

The E. coli BL21 (DE3) and E. coli DH5a cells used in the experiments of this project were obtained from Dr. SuiLam Wong and Dr. Richard Moore of University of Calgary, respectively. The protocol used to transform both strains of E. Coli was provided by Dr. Richard Moore.

The strain to be transformed was cultured in $2 \mathrm{~mL} L B$ broth and shaken at $28^{\circ} \mathrm{C}$ overnight. The strained was then subcultured 1:50 into $50 \mathrm{~mL} \mathrm{LB}$ with $10 \mathrm{mM} \mathrm{MgSO} 4$ and $1 \mathrm{mM} \mathrm{KCl}$ and shaken at $28^{\circ} \mathrm{C}$ until OD600 reaches 0.3 to 0.4. After chilling on ice for at least 10 minutes, the mixture was transferred to a $50 \mathrm{~mL}$ pre-chilled tube. The mixture was then centrifuged at $2,500 \mathrm{~g}$ for 8 minutes at $4{ }^{\circ} \mathrm{C}$ and was resuspended in $10 \mathrm{~mL}$ ice-cold $100 \mathrm{mM} \mathrm{CaCl}$. The cells were gently mixed on ice and incubated on ice, for a period of time between 10 minutes to overnight. Then, the tube of cells was centrifuged at $2500 \mathrm{xg}$ for 8 minutes at $4^{\circ} \mathrm{C}$ and resuspended in $500 \mu \mathrm{L} 100 \mathrm{mM} \mathrm{CaCl} 2$ and $10 \%$ glycerol. The mixture was mixed by gently pipetting up and down and left on ice for at least 10 minutes. The cells were then separated to $1.5 \mathrm{~mL}$ pre-chilled tubes as $50 \mu \mathrm{L}$ aliquots, while remaining on ice.

The competency of the cells was then assessed by transformation with pUC19 from NEB. $50 \mu \mathrm{L}$ of competent cells and $1 \mu \mathrm{L} \mathrm{pUC19}(50 \mathrm{pg} / \mu \mathrm{L})$ were combined and left on ice for 40 minutes. The mixture was then put into a water bath at $37^{\circ} \mathrm{C}$ for $45 \mathrm{~s}$. On ice, $400 \mu \mathrm{L}$ of LB were added and shaken for 1 hour at $37^{\circ} \mathrm{C} .225 \mu \mathrm{L}$ of the culture was then streaked on an ampicillin $(100 \mu \mathrm{g} / \mathrm{mL})$ plate and grown overnight at $37^{\circ} \mathrm{C}$.

\section{Cloning}

The IDT geneBlocks were digested and ligated to pET29b(+). The restriction enzymes used are given in Table 1. After the genes were inserted in the plasmid, E. coli DH5a was transformed with the vector. DH5a strain was used because of its ability to produce a high copy number of plasmids. Plasmid miniprep was carried out and the obtained plasmids were digested and run on gel electrophoresis to confirm if ligation was successful.

Table 1. Restriction enzymes used for digestion of pET29b(+) and the respective genes.

\section{Gene Restriction Enzymes}

phaBA HindlII, Kpnl

phaC Notl, HindlII

phaCJ Notl, HindIII 
This work was assessed during the iGEM/PLOS Realtime Peer Review Jamboree on $23^{\text {rd }}$ February 2018 and has been revised in response to the reviewers' comments. A transcript of these comments is available with the original article as a supplementary file

phaP-hlyA EcoRI, Spel

After cloning the genes given in Table 1, some of the genes were ligated together. Digested phaBA and phaC vectors were excised from low-melting point gel and ligated together followed by transformation of $E$. coli $\mathrm{DH} 5 a$ with the resulting plasmid. The restriction enzymes used were Xbal and HindIII. Standard protocols for digestion, ligation, and transformation were followed. After confirmation whether the genes were successfully ligated, the final operons (phaCBA and phaCJ) were sequenced.

After cloning the genetic constructs in $E$. coli DH5a and confirmation of the gene sequences, plasmid miniprep was carried out. The obtained plasmids were then used to transform E. coli BL21 (DE3) because of this strain's ability to synthesize more protein. Hence, all genetic constructs were cloned in E. coli BL21 (DE3), which was used for experiments.

\section{Digestion of constructs and vectors}

In order to carry out a restriction digest, DNA of known concentration was obtained. In a $1.5 \mathrm{~mL}$ microcentrifuge tube, $1 \mu \mathrm{g}$ DNA suspended in TE buffer or ddH2O was added. For a $20 \mu \mathrm{L}$ solution, $1 \mu \mathrm{L}$ of the desired restriction enzyme(s) was added to the tube. $2 \mu \mathrm{L}$ of $10 x$ buffer for the respective enzyme was added. Finally, ddH2O was added to the tube to adjust final volume to $20 \mu \mathrm{L}$. The tube was then allowed to incubate at $3^{\circ} \mathrm{C}$ for an hour followed by heat inactivation of the restriction enzymes by placing the tube in water bath at $80^{\circ} \mathrm{C}$ for 20 minutes. The tube was then stored in $-20^{\circ} \mathrm{C}$.

The restriction enzymes used for our experiments were from New England Biolabs (NEB) and the manufacturer's protocol was used for the respective restriction enzymes.

\section{Ligation of Vector and DNA insert}

In order to clone the respective DNA used for experiments, the DNA parts were ligated to a plasmid backbone (pSB1C3 or pET29b(+)). The vector was then used to transform E. coli DH5a for plasmid propagation or E.coli BL21(DE3) for protein expression. The protocol used for ligation experiments were obtained from NEB. In a $1.5 \mathrm{~mL}$ microcentrifuge tube, $50 \mathrm{ng}$ of the digested vector DNA suspended in TE buffer or ddH2O was added. For a $20 \mu \mathrm{L}$ reaction, $2 \mu \mathrm{L}$ of 10x T4 DNA ligase buffer was added to the tube. The DNA insert:vector ratio used was 3:1 and the desired amount of plasmid was calculated and the appropriate volume of vector was added to tube. $1 \mu \mathrm{L}$ of T4 DNA ligase was added to tube followed by incubation of tube on ice placed at room temperature overnight. Finally, the tube was stores at $-20^{\circ} \mathrm{C}$ for use in further experiments.

\section{Plasmid Miniprep}

Plasmid miniprep was carried out to analyze the DNA. Cells were cultured in culture tubes containing LB and the respective antibiotic. $2 \mathrm{~mL}$ of the overnight culture was transferred by pipetting into a $2 \mathrm{~mL}$ microcentrifuge tube. The microcentrifuge tube was spun at $3500 \times$ g for 10 minutes and a cell pellet was obtained. The supernatant was discarded. Cells were then resuspended in $300 \mu \mathrm{L}$ resuspension buffer. $300 \mu \mathrm{L}$ of lysis buffer was added and the tube was inverted gently. The tube was left to incubate at $23^{\circ} \mathrm{C}$ for $3-5$ minutes. $300 \mu \mathrm{L}$ of precipitation buffer was added and tube was inverted gently. The resulting white suspension was centrifuged at 14,000xg for 10 minutes at $23^{\circ} \mathrm{C}$. The supernatant obtained was transferred to a clean $1.5 \mathrm{~mL}$ microcentrifuge tube using pipette. $650 \mu \mathrm{L}$ of isopropanol was added and tube was gently inverted. It was then allowed to incubate at $23^{\circ} \mathrm{C}$ for 10 minutes. The 
This work was assessed during the iGEM/PLOS Realtime Peer Review Jamboree on $23^{\text {rd }}$ February 2018 and has been revised in response to the reviewers' comments. A transcript of these comments is available with the original article as a supplementary file

tube was placed in microcentrifuge and spun at 14,000xg for 10 minutes at $4^{\circ} \mathrm{C}$. The resulting supernatant was discarded and pellet was washed with $500 \mu \mathrm{L}$ cold $70 \%$ ethanol. The tube was placed in micro Centrifuge at $14,000 \times$ for 5 minutes at $4^{\circ} \mathrm{C}$ and supernatant was discarded. The pellet was dried by placing microcentrifuge in speed vac for 15-30 minutes, or until no more liquid was visible in the tube. The pellet was suspended in $\mathrm{ddH}_{2} \mathrm{O}$ and stored at $-20^{\circ} \mathrm{C}$.

\section{Bacterial Transformation}

In order to test our genetic constructs, E. coli BL21(DE3) was transformed with the respective genes inserted in pET29b(+) or pSB1C3. $50 \mu \mathrm{L}$ aliquot of competent E.coli BL21(DE3) cells were obtained from $-80 \mathrm{C}$ freezer and placed on ice to thaw just before use. 0.3-1 $\mu \mathrm{g}$ of DNA was added to cells using pipette and the microcentrifuge tube containing the cells was gently flicked to allow DNA to mix with cells. $\mathrm{CaCl}_{2}$ concentration was adjusted to maintain competency of the cells and the mixture was incubated on ice for 45 minutes. The cells were then heat shocked for $60-75$ seconds at $42^{\circ} \mathrm{C}$. The microcentrifuge tube was placed on ice for 5 minutes. $2 \mathrm{~mL}$ of SOC medium was added to the aliquot of cells followed by incubation for $60-90$ minutes at $37^{\circ} \mathrm{C}$, shaking at $200 \mathrm{rpm} .50-100 \mu \mathrm{L}$ of resuspended culture was obtained and plated on agar plate with appropriate antibiotic. The cells were spread using aseptic technique. Finally, the agar plate was placed in incubator set to $37^{\circ} \mathrm{C}$ overnight or until desired growth was observed.

\section{Confirmation Digest}

After transforming $E$. coli with the desired vector containing gene of interest, a confirmation digest was carried out to analyze the presence of DNA insert in the vector. The four conditions used for the confirmation digest were: (i) Plasmid of interest containing vector digested with restriction enzymes used for ligation, (ii) Plasmid with no insert digested with restriction enzymes used for ligation, (iii) Plasmid of interest containing vector digested with restriction enzyme that cuts at the region of DNA insert and another enzyme that cuts the backbone, and (iv) Plasmid with no insert digested with restriction enzyme that cuts at the region of DNA insert and another enzyme that cuts the backbone. Finally, the product of the reaction was analyzed using agarose gel electrophoresis. A $1 \%$ agarose gel was run at $100 \mathrm{~V}$ for 30 minutes.

\section{PHB Synthesis}

\section{Utilizing Acetyl-CoA to produce PHB}

Synthetic feces (see below for Preparation of Synthetic Feces and Supernatant) were fermented to produce acetylCoA for PHB synthesis. After fermentation of synthetic feces, glucose is converted to acetic acid followed by acetylCoA formation. To test whether phaCBA construct can synthesize PHB using fermented synthetic feces, three replicates of overnight cultures having OD600 $=0.4-0.8$ of each negative control (vector with no insert) and vector with phaCBA insert were made. The overnight cultures were grown in LB media and the respective antibiotic (kanamycin, $50 \mu \mathrm{g} / \mathrm{mL}$ ). In $125 \mathrm{~mL}$ Erlenmeyer flasks, $10 \mathrm{~mL}$ overnight cultures were added followed by the addition of $10 \mathrm{~mL}$ fermented synthetic feces, $100 \mathrm{uL} \mathrm{MgSO} 4(2 \mathrm{mM}), 5 \mathrm{uL} \mathrm{CaCl} 2(0.1 \mathrm{mM}), 10 \mathrm{ml} \mathrm{M} 9$ salts $(1 \mathrm{x})$, and $5 \mathrm{uL} 1 \mathrm{M}$ IPTG. Final volume in flasks were adjusted to $50 \mathrm{~mL}$ with ddH2O. The flasks were covered with aluminum foil and placed in a shaker for 24 hours at $37^{\circ} \mathrm{C}$ and $100 \mathrm{rpm}$. Solutions in the flasks were obtained next day and poured into $50 \mathrm{~mL}$ Falcon ${ }^{\mathrm{TM}}$ tubes. Chemical lysis was carried out using sodium hypochlorite extraction to analyze PHB production. 
This work was assessed during the iGEM/PLOS Realtime Peer Review Jamboree on $23^{\text {rd }}$ February 2018 and has been revised in response to the reviewers' comments. A transcript of these comments is available with the original article as a supplementary file

\section{Utilizing VFAs to produce PHB}

Other VFAs present in fermented synthetic feces are propionic acid, butyric acid, etc. Beta-oxidation pathway uses the VFAs and phaCJ construct can be used to convert the products to produce PHB in bacteria. Thus, four different conditions were used to test whether PHB is synthesized using fermented synthetic feces: negative control (vector with no insert), positive control (vector with insert and glucose present in media), pure VFAs, and fermented synthetic feces. Pure VFAs were used to test how much PHB is produced by utilizing VFAs when no glucose is present. 9 replicates of overnight cultures of $E$. coli BL21(DE3) transformed with $\mathrm{pET} 29 \mathrm{~b}(+)$ containing phaCJ insert were made along with 3 replicates of overnight cultures of the negative control (pET29b(+) with no insert). OD600 of the overnight cultures was adjusted to lie between 0.4 and $0.8 .10 \mathrm{~mL}$ of the cultures were added to $125 \mathrm{~mL}$ Erlenmeyer flasks. $100 \mathrm{uL} \mathrm{MgSO} 4(2 \mathrm{mM}), 5 \mathrm{uL} \mathrm{CaCl} 2(0.1 \mathrm{mM}), 10 \mathrm{ml}$ M9 salts (1x), and $5 \mathrm{uL} 1 \mathrm{M}$ IPTG were added to each flask.

$7 \mathrm{~mL}$ of $20 \%$ glucose solution was added to the positive control. Propionic acid, acetic acid, and butyric acid were in 3:2:1 ratio to the pure VFAs condition. Lastly, $10 \mathrm{~mL}$ of fermented synthetic feces was added to each of the remaining replicates. The flasks were covered with aluminum foil and placed in a shaker for 24 hours at $37^{\circ} \mathrm{C}$ and $100 \mathrm{rpm}$. The cultures were obtained the next day and poured into $50 \mathrm{~mL}$ Falcon ${ }^{\mathrm{TM}}$ tubes. The tubes were centrifuged, and the resulting supernatant was discarded. Cell pellet was resuspended in $1 \mathrm{x}$ PBS for extraction and the respective OD600 were recorded before proceeding to sodium hypochlorite extraction.

\section{Sodium Hypochlorite Extraction of PHB}

Sodium hypochlorite (bleach) was used to chemically lyse the bacterial cells, causing them to release PHB into their media, which can then be isolated via centrifugation. This extraction method of carried out for simplistic analysis of PHB production. $50 \mathrm{~mL}$ of PHB-producing E. coli BL21(DE3) overnight cultures were centrifuged at 3275xg for 10 minutes in a $50 \mathrm{~mL}$ Falcon ${ }^{\mathrm{TM}}$ Tube. The supernatant was discarded, and the pellet was resuspended in $5 \mathrm{~mL} 1 \mathrm{X}$ PBS solution. The resuspended mixture was centrifuged again at $3275 \mathrm{xg}$ for 10 minutes. The supernatant was discarded, and the pellet was resuspended in $5 \mathrm{~mL} 1 \%(\mathrm{v} / \mathrm{v})$ Triton X-100 in PBS and left to incubate for 30 minutes at $23^{\circ} \mathrm{C}$. The mixtures were centrifuged at $3275 \mathrm{xg}$ for 10 minutes. The supernatant was discarded. The pellet was resuspended in $5 \mathrm{~mL} 1 \mathrm{X}$ PBS solution and centrifuged at $3275 \mathrm{xg}$ for 10 minutes. The supernatant was discarded. The mixture was then resuspended in $5 \mathrm{~mL}$ of sodium hypochlorite and left to incubate at $30^{\circ} \mathrm{C}$ for 1 hour. After the hour, the mixture was centrifuged at $3275 \mathrm{xg}$ for 20 minutes. The supernatant was discarded, and the pellet was washed in $5 \mathrm{~mL} 70 \%$ ethanol. This wash step was repeated several times, with 20 minutes of centrifugation at $3275 x g$ between each step. The extracted PHB powder was then allowed to dry overnight in an open tube.

\section{PHB Secretion}

E. coli BL21(DE3) transformed with phaCAB-phasin-HlyA in a pSB1C3 plasmid backbone was used to test the production and secretion of PHB into the extracellular media. E. coli BL21(DE3) transformed with phaCAB in a pSB1C3 plasmid backbone was used as a negative control. All experiments were carried out in triplicates. $1 \mathrm{~mL}$ of overnight culture was inoculated in $49 \mathrm{~mL}$ LB media $+3 \%$ glucose $+30 \mu \mathrm{g} / \mathrm{mL}$ chloramphenicol (total volume of $50 \mathrm{~mL}$ ), induced with $5 \mu \mathrm{L} 1 \mathrm{M} \mathrm{IPTG}$, then incubated at $37^{\circ} \mathrm{C}$ and $150 \mathrm{rpm}$ in an aerobic environment for 48 hours. At 24-hour intervals, the culture was transferred to a $50 \mathrm{~mL}$ Falcon ${ }^{\mathrm{TM}}$ tube and $500 \mu \mathrm{L}$ of $1 \mathrm{M} \mathrm{CaCl} 2(0.05549 \mathrm{~g} \mathrm{CaCl} 2)$ was added to to the cultures to reach a final concentration of $0.01 \mathrm{M} \mathrm{CaCl} 2$. After 10 minutes incubation at $23^{\circ} \mathrm{C}$, the solution was centrifuged at $50 \mathrm{xg}$ for 5 minutes and the supernatant removed to a new $50 \mathrm{~mL} \mathrm{Falcon}{ }^{\mathrm{TM}}$ tube. This pellet contains the secreted fraction of PHB and cellular debris. The supernatant was centrifuged at $3270 \times \mathrm{xg}$ for 10 
This work was assessed during the iGEM/PLOS Realtime Peer Review Jamboree on $23^{\text {rd }}$ February 2018 and has been revised in response to the reviewers' comments. A transcript of these comments is available with the original article as a supplementary file

minutes and the supernatant discarded. This pellet contains the intracellular PHB granules inside live cells, which were not secreted.

To remove cellular debris, the pellet of secreted PHB was resuspended in $5 \mathrm{~mL}$ of $1 \%$ Triton X-100 in PBS, incubated for 30 minutes at $23^{\circ} \mathrm{C}$, then Centrifuged at $3275 \mathrm{~g}$ for 10 minutes. The supernatant was discarded and the pellet was dried overnight in an open tube at $23^{\circ} \mathrm{C}$. To extract $\mathrm{PHB}$ from the intracellular fraction, the pellet was treated with sodium hypochlorite and dried overnight in an open tube at $23^{\circ} \mathrm{C}$. This protocol was adapted from Rahman et al. 2013.

\section{Characterization of PhaJC and PhaCAB}

\section{SDS-PAGE}

Overnights cultures of $E$. coli containing the pet29b(+), pet29b(+)-phaCJ, and pet29b(+)-phaCBA vector were made and left to incubate at $37^{\circ} \mathrm{C}$ in a shaker for $16 \mathrm{hrs}$ at $200 \mathrm{rpm} .100 \mu \mathrm{L}$ of the overnight cultures were then subcultured in a new tube of $2 \mathrm{~mL}$ LB for $2 \mathrm{hr}$. The bacteria were then induced with $0.1 \mathrm{mM}$ IPTG and left in the shaker for another 4 hours. The cultures were then pelleted in microcentrifuge tubes spun at max. The pellets were put in the $-20^{\circ} \mathrm{C}$ freezer and left overnight. The next day, the pellet was thawed at room temperature. The pellet was resuspended in $100 \mu \mathrm{L}$ of STET buffer. $1 \mathrm{mg}$ of lysozyme was added to the resuspension and left to incubate in a water bath at $37^{\circ} \mathrm{C}$ for 15 minutes. The suspensions were then sonicated for 5 seconds on and 5 seconds off while on ice. Centrifugation was used to separate the soluble and insoluble proteins by spinning the sonicated suspensions at max for $10 \mathrm{~min}$. The soluble portion was pipetted into another microcentrifuge tube. The insoluble pellet was resuspended in 50 $\mathrm{L}$ loading buffer. Protein loading dye was made by combining $50 \mu \mathrm{L}$ of loading buffer with $3 \mu \mathrm{L}$ DTT. $15 \mathrm{uL}$ of the soluble and insoluble protein fractions were aliquoted into microcentrifuge tubes. $3 \mathrm{uL}$ of the loading dye was added to each protein sample. The protein samples with the dye were boiled for 5 minutes to denature the proteins and then ran on a 1 X SDS-PAGE gel for $30 \mathrm{mAmp}$ for 30-40 minutes.

The materials used for the gel and procedures for setting up an SDS-PAGE gel are listed below.

$1 \times$ SDS gel loading buffer

. $\quad 50 \mathrm{mM}$ tris- $\mathrm{Cl}(\mathrm{pH} 6.8)$

. $100 \mathrm{mM}$ dithiothreitol

- $2 \%$ sodium dodecyl sulfate

- $0.1 \%$ bromophenol blue

- $10 \%$ glycerol

1x Tris-Glycine electrophoresis buffer:

. $25 \mathrm{mM}$ tris

- $250 \mathrm{mM}$ glycine

- $0.1 \%(w / v)$ sodium dodecyl sulfate

Stacking gel:

- $\mathrm{dH}_{2} \mathrm{O}$

- $30 \%$ acrylamide mix

- $1.0 \mathrm{M}$ tris $(\mathrm{pH} 6.8)$

- $10 \%$ sodium dodecyl sulfate 
This work was assessed during the iGEM/PLOS Realtime Peer Review Jamboree on $23^{\text {rd }}$ February 2018 and has been revised in response to the reviewers' comments. A transcript of these comments is available with the original article as a supplementary file

. $10 \%$ ammonium persulfate

. TEMED

$10 \%$ Resolving gel:

. $\mathrm{dH}_{2} \mathrm{O}$

- $30 \%$ acrylamide mix

. $1.5 \mathrm{M}$ tris $(\mathrm{pH} 8.8)$

. $10 \%$ sodium dodecyl sulfate

- $10 \%$ ammonium persulfate

- TEMED

HPLC PHB quantification was conducted using high performance liquid chromatography (HPLC) by digesting samples in concentrated acid to produce crotonic acid. The protocol was based on the methodology outlined in [16]. PHB-containing samples were first digested in $1 \mathrm{ml}$ concentrated sulphuric acid in a 90 degrees Celsius water bath for 30 minutes. After the digestion was complete the samples were cooled on ice and $4 \mathrm{ml}$ of $0.014 \mathrm{~N}$ sulphuric acid was rapidly mixed in. Samples were diluted using $0.014 \mathrm{~N}$ sulphuric acid, with dilution factors ranging from 10-100. The samples were filtered through a 0.2 micron filter before running them on a Aminex HPX-87H ion-exclusion HPLC column to measure crotonic acid. PHB content in the samples was calculated by assuming an $60 \%$ conversion, which was found by digesting known concentrations of standard industrial-grade PHB samples.

\section{PHB Extraction}

\section{Chemical coagulation of PHB}

One of the design options we considered for PHB extraction used the principle of chemical coagulation. In order to test chemical coagulation of PHB particles we first simulated nanoscale PHB, which was the expected size range of secreted PHB [9]. $5 \mathrm{~g} / \mathrm{L}$ industrial-grade PHB in suspension in distilled water was sonicated in $250 \mathrm{ml}$ batches in a probe-type sonicator to break up the particles. The suspension was then allowed to settle for 48 hours before the top phase was separated by decanting. Three conditions were tested with replicates to show that adding a coagulant helped the nanoparticles to agglomerate into larger particles that could be more easily recovered by centrifugation. The test conditions included, centrifugation at 1000 RPM, centrifugation at 3750 RPM, and centrifugation at 3750 RPM after making up a $10 \mathrm{mM}$ calcium chloride concentration in the sample. Amount of PHB extracted was estimated by measuring the absorbance of each of the sample at $600 \mathrm{~nm}$.

\section{Electrocoagulation of PHB}

We also tested electrocoagulation to extract PHB nanoparticles from a suspension in distilled water and from synthetic feces supernatant. Supernatant from the synthetic feces recipe was prepared by centrifuging samples in $50 \mathrm{ml}$ tubes at $3750 \mathrm{RPM}$ for 20 minutes, removing the supernatant and then centrifuging the supernatant again at 3750 RPM for 20 minutes. $5 \mathrm{~g} / \mathrm{L}$ suspension of PHB in distilled water was prepared by sonication the suspension in $250 \mathrm{ml}$ batches in a probe-type sonicator. The suspension was then allowed to settle for 48 hours before the top phase was separated by decanting. The electrocoagulation cell was prepared by connecting an iron anode and a steel cathode to a 9 volt battery and placing the test sample in the cell [17]. Three types of samples were tested: PHB suspension in distilled water to demonstrate whether PHB particles could be made to settle using electrocoagulation, synthetic feces supernatant as a negative control, and a 1:1 mixture of synthetic feces 
This work was assessed during the iGEM/PLOS Realtime Peer Review Jamboree on $23^{\text {rd }}$ February 2018 and has been revised in response to the reviewers' comments. A transcript of these comments is available with the original article as a supplementary file

supernatant and PHB suspension to test whether electrocoagulation could work to separate PHB from feces supernatant. The last test sample simulated the real mixture PHB would have to be separated from in the process designed for Mars. Each cell was run for 3 hours after $250 \mathrm{ml}$ of the test sample was loaded into the cell. Qualitative results were obtained by observing the settlement.

\section{Preparation of Synthetic Feces and Supernatant}

Due to safety and ethical concerns with using human feces, synthetic feces mimicking the chemical and physical properties of real feces were used for the experiments. In addition to water, human feces contain fats, carbohydrates, nitrogenous material, minerals, and bacterial debris. Synthetic feces were prepared using a modified recipe from National Aeronautics and Space Administration (NASA). The original recipe included water, dry baker's yeast, microcrystalline cellulose, psyllium, miso paste, oleic acid, sodium chloride, potassium chloride and calcium chloride (Table 2).

The recipe was modified to include yeast extract in place of dry baker's yeast to avoid fermentation of synthetic feces by yeast and oleic acid was removed from the recipe due to challenges with processing samples containing oils on the HPLC (Table 3).

Table 2. Original NASA recipe for preparation of synthetic feces [18].

\section{Compound}

Water

Baker's yeast (dry)

Microcrystalline cellulose

Psyllium

Miso paste

Oleic acid

$\mathrm{NaCl}$

$\mathrm{KCl}$

$\mathrm{CaCl} 2$

\section{Amount (g per kg feces)}

800

60

30

35

35

40

4

4

2

Table 3. Modified recipe used for preparation of synthetic feces

\section{Compound}

Water

Yeast extract

Microcrystalline cellulose

Psyllium

\section{Amount (g per kg feces)}

800

60

35 
This work was assessed during the iGEM/PLOS Realtime Peer Review Jamboree on $23^{\text {rd }}$ February 2018 and has been revised in response to the reviewers' comments. A transcript of these comments is available with the original article as a supplementary file

Miso paste

$\mathrm{NaCl}$

$\mathrm{KCl}$

$\mathrm{CaCl} 2$
35

4

4

2

Some experiments required synthetic feces supernatant, where solid particles were removed. To obtain synthetic feces supernatant, synthetic feces were centrifuged at $3000 \mathrm{rcf}$ for $20 \mathrm{~min}$. The liquid portion was then collected and sterilized using $0.20 \mu \mathrm{m}$ syringe filters. The solid portion was discarded. The supernatant was stored at $-20^{\circ} \mathrm{C}$, if required.

\section{Effect of Temperature on VFA Fermentation}

Bench scale experiments were performed to determine the optimal operating temperature for the VFA fermentation step of the process. The two temperatures selected for the experiment were $37^{\circ} \mathrm{C}$ and $22^{\circ} \mathrm{C}$ corresponding to the optimal bacterial fermentation temperature and expected room temperature in a Mars habitat, respectively. Synthetic feces were fermented with E. coli BL21(DE3) transformed with $\mathrm{pET} 29 \mathrm{~b}(+)$ vector without any inserts (control), PHB-producing E. coli transformed with a PHB-producing part from iGEM Imperial College 2013 ("Imperial condition") and PHB-producing E. coli transformed with PHB-producing part from iGEM Tokyo 2012 ("Tokyo condition") inside a shaker at $80 \mathrm{rpm}$ and desired temperature. Different fermentation durations were tested including 3 and 5 days. Synthetic feces were inoculated with $1 \mathrm{~mL}$ of overnight $E$. coli cultures per $100 \mathrm{~mL}$ of synthetic feces. Erlenmeyer flasks used for fermentation were sterilized before the experiment and were covered with aluminum foil during fermentation. After fermentation, fermented synthetic feces were centrifuged at 3000 rcf for 20 minutes. The supernatant was then collected. A sample of the supernatant was taken for VFA measurements using titrations, while the remaining supernatant was sterilized using $0.20 \mu \mathrm{m}$ syringe filters. Sterilized supernatant was then fermented with PHB-producing bacteria, as previously described. The amounts of PHB produced from each VFA fermentation condition were then compared.

\section{VFA Quantification using Titrations}

A titration method described by Anderson and Yang (1992) was used to quantify VFA concentration in synthetic poop supernatant. To quantify VFA, $50 \mathrm{~mL}$ of diluted sample were placed in a $100 \mathrm{~mL}$ beaker [19]. The beaker was placed on the magnetic stir plate and a magnetic stir bar was used to continuously mix the sample during titrations. $\mathrm{A} \mathrm{pH}$ probe was placed into the solution to measure the change in $\mathrm{pH}$. After recording the initial $\mathrm{pH}, 1 \mathrm{~N}$ sulfuric acid was added in $100 \mu \mathrm{L}-1 \mathrm{~mL}$ increments until a set point of 5.1 followed by 3.5 . The volume of acid titrated to reach the desired $\mathrm{pH}$ set point was recorded. The following equations were used to calculate the concentration of VFA: $A 1=[V F A][H 2]-[H 1][H 2]+k 1 \quad$ Equation 1

$\mathrm{A} 2=[\mathrm{VFA}][\mathrm{H} 3]-[\mathrm{H} 1][\mathrm{H} 3]+\mathrm{k} 2 \quad$ Equation 2

Where $A 1=$ molar equivalent of acid consumed to the first titration point

$\mathrm{A} 2=$ molar equivalent of acid consumed to the second titration point 
This work was assessed during the iGEM/PLOS Realtime Peer Review Jamboree on $23^{\text {rd }}$ February 2018 and has been revised in response to the reviewers' comments. A transcript of these comments is available with the original article as a supplementary file

[H1],[H2], [H3] = concentration of $\mathrm{H}+$ ions initially and at the two titration points

$[\mathrm{VFA}]=$ concentration of VFA

$\mathrm{k} 1=$ conditional dissociation constant for carbonic acid

$\mathrm{k} 2=$ combined dissociation constant for VFA

Equations above can be rearranged to solve for VFA concentration.

\section{Equivalent System Mass Analysis}

Equivalent System Mass (ESM) analysis, a method recommended by NASA to evaluate advanced life support systems, was used to evaluate the feasibility of the proposed process and to compare different process options [20']. ESM analysis is used to identify which of the proposed designs has the lowest launch cost based on mass, volume, power, cooling, and crewtime needs in place of traditional dollar costs. The following formula was used to determine ESM value for the proposed process:

$\mathrm{ESM}=\mathrm{M}+\mathrm{IMd}+(\mathrm{V}+\mathrm{IVd}) \mathrm{Veq}+\mathrm{PPeq}+\mathrm{PtPt}$ eq Equation 3

Where $\mathrm{ESM}=$ equivalent system mass $(\mathrm{kg})$

$\mathrm{M}=$ mass of the system $(\mathrm{kg})$

$\mathrm{IM}=$ logistics mass $(\mathrm{kg} /$ day $)$

$\mathrm{d}=$ mission duration (days)

$\mathrm{V}=$ volume of the system $(\mathrm{m} 3)$

IV = logistics volume (m3/ day)

Veq= volume equivalency factor $(\mathrm{kg} / \mathrm{m} 3)$

$\mathrm{P}=$ power requirement of the system $(\mathrm{kW})$

$\mathrm{Peq}=$ power equivalency factor $(\mathrm{kg} / \mathrm{kW})$

$\mathrm{Pt}=$ thermal control power requirement $(\mathrm{kW})$

Pt eq= thermal control equivalency factor $(\mathrm{kg} / \mathrm{kW})$

The crew time requirements were not considered in the analysis due to lack of data. ESM equivalency factors were obtained from NASA's Baseline Values and Assumptions for Advanced Life Support Systems [21] and are summarized in Table 4.

Table 4. Equivalency factors for volume and power used in ESM analysis of the process

\section{ESM Equivalency Factor Units Nominal}

Shielded volume $\quad \mathrm{kg} / \mathrm{m} \quad 3216.5$

Unshielded volume $\mathrm{kg} / \mathrm{m} \quad 39.16$

$\begin{array}{lll}\text { Power } & \mathrm{kg} / \mathrm{kW} & 87\end{array}$

Thermal control $\quad \mathrm{kg} / \mathrm{kW} \quad 146$ 
This work was assessed during the iGEM/PLOS Realtime Peer Review Jamboree on $23^{\text {rd }}$ February 2018 and has been revised in response to the reviewers' comments. A transcript of these comments is available with the original article as a supplementary file

\section{Process Development Assumptions}

A number of assumptions were made based on Anderson et al. (2015) when developing the physical process that starts with feces and end with a bioplastic powder that can be used in a 3D printer [21]. The nominal surface habitat duration was assumed as 600 days with a crew size of 6 people. The process was designed to accommodate a maximum of $150 \mathrm{~g}$ of fecal matter per crew member per day corresponding to a volume of $150 \mathrm{~mL}$ per crew member per day. Based on the amount of fecal matter, the predicted water recovery for VFA and PHB production was assumed to be $123 \mathrm{~g}$ or $123 \mathrm{~mL}$ per crew member per day. The power required for the process will be supplied using solar panels and nuclear sources, which were assumed to be available on Mars.

\section{Results}

\section{Successful cloning of phaCJ and phaCBA}

A double digest confirmation was carried out to confirm the presence of phaCJ insert in pET29b vector. The digestion utilized Notl and Hindlll restriction enzymes. The length of phaJ is $474 \mathrm{bp}$. Hence, the total sum of phaC and phaJ genes is around $2.2 \mathrm{~kb}$. The products of confirmation digest were analyzed through agarose gel electrophoresis. The results showed the presence of two linear DNA of sizes $5.4 \mathrm{~kb}$ and $2.2 \mathrm{~kb}$. The different lanes show results of confirmation digest on plasmid miniprep of cell cultures prepared from different colonies, which were obtained after transformation of $E$. coli.

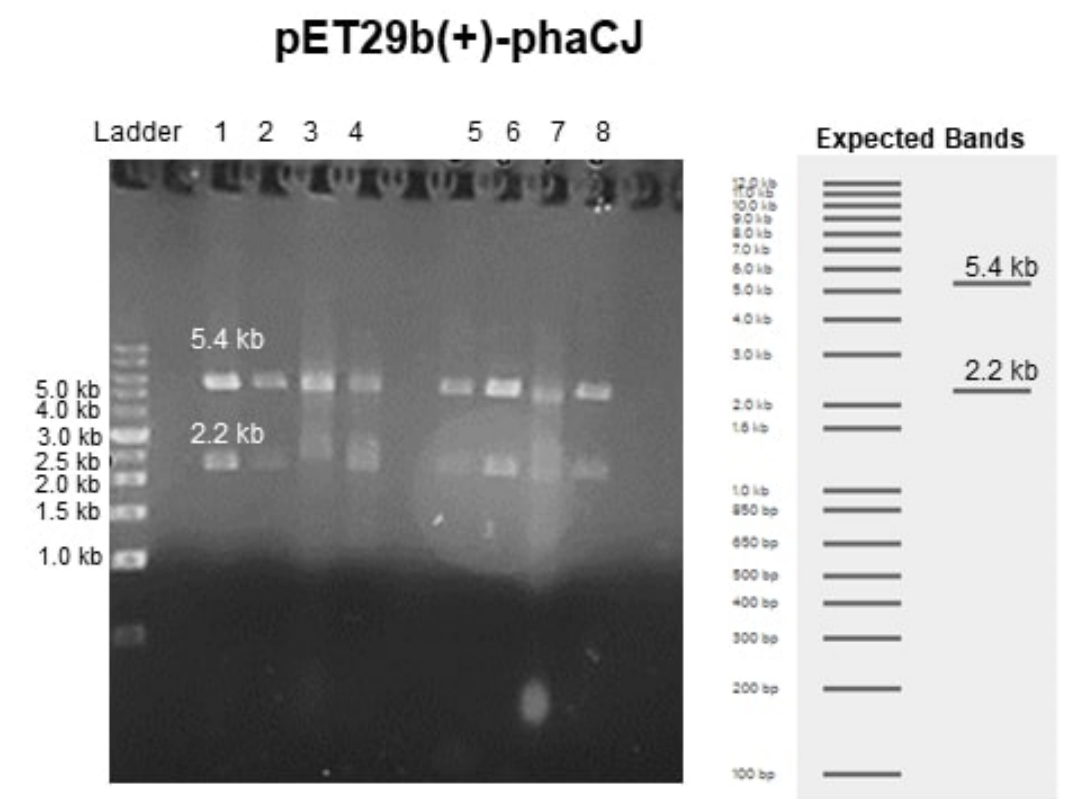

Fig 1. pet29b(+)-phaCJ plasmid digested with Notl and HindIII ran on $1 \%$ agarose gel.

The pet29b(+) vectors containing phaCBA insert were analyzed through gel electrophoresis after performing digestion confirmation. Fig 2. shows the results of digesting pET29b vector containing phaCBA operon using Kpnl and Notl. The length of phaC, phaB, and phaA genes are $1.8 \mathrm{~kb}, 759 \mathrm{bp}$, and $1.2 \mathrm{~kb}$. The products of the confirmation digest were analyzed through agarose gel electrophoresis. The results showed the presence of DNAs of length $5.3 \mathrm{~kb}, 2.8 \mathrm{~kb}$, and $1.0 \mathrm{~kb}$ respectively. The length of pET29b vector is around $5.4 \mathrm{~kb}$ and the sum of the length of phaCBA operon is $3.8 \mathrm{~kb}$. The sum of the two lower bands was observed to be $3.8 \mathrm{~kb}$. 


\section{pET29b(+) -phaCBA}

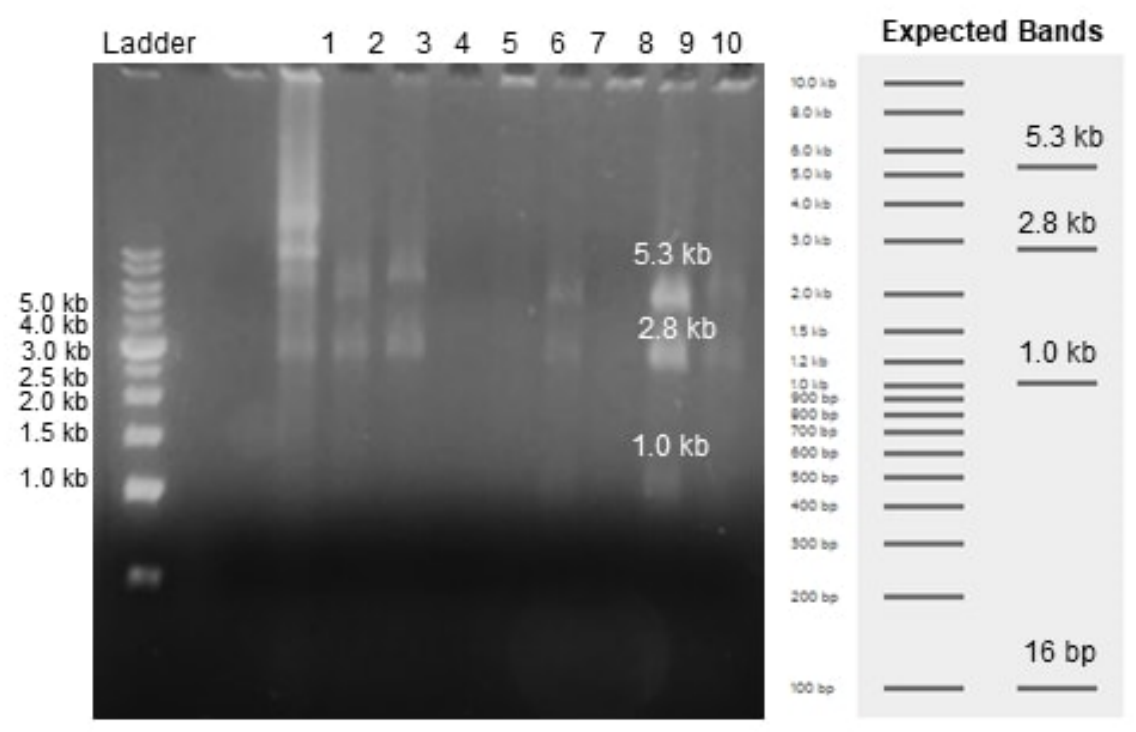

Fig 2. phaCBA was successfully cloned into the pet29b(+) vector $E$. coli. Double digest confirmation of pet29b(+)-phaCBA with Notl and Kpnl ran on $1 \%$ agarose gel.

An SDS-PAGE gel was ran to determine whether the E. coli cloned with the vectors containing phaCJ and phaCBA could produce the proteins of interest. $0.1 \mathrm{mM}$ IPTG was added to a $2 \mathrm{~mL}$ subculture of overnight culture cultivated for $16 \mathrm{~h}$ and further cultivated for $2 \mathrm{~h}$. The soluble and insoluble protein fractions were loaded in the lanes outlined in Fig 3. phaJ and phaC activity was detected in the insoluble protein fraction. A dark band was observed in the pET29b(+)-phaCJ lane between 17-22 kDa, which is in reasonable agreement with the approximate mass of the predicted PhaJ product (17.7kDa). Another protein was observed in the insoluble portion of pET29b(+)-phaCBA, which is in reasonable agreement with the mass of the predicted PhaA product $(41.4 \mathrm{kDa})$. These protein bands were not seen in the control pET29b(+) lanes. The protein bands of the PhaC $(65.1 \mathrm{kDa})$ and PhaB (27.2) for pET29b(+)-phaCBA were not observed. The regions where the expected protein bands for PhaC from pet29b(+)phaCJ and PhaC and PhaB from pet29b(+)-phaCBA were smeared, unclear, and had many close bands together. 
This work was assessed during the iGEM/PLOS Realtime Peer Review Jamboree on $23^{\text {rd }}$ February 2018 and has been revised in response to the reviewers' comments. A transcript of these comments is available with the original article as a supplementary file

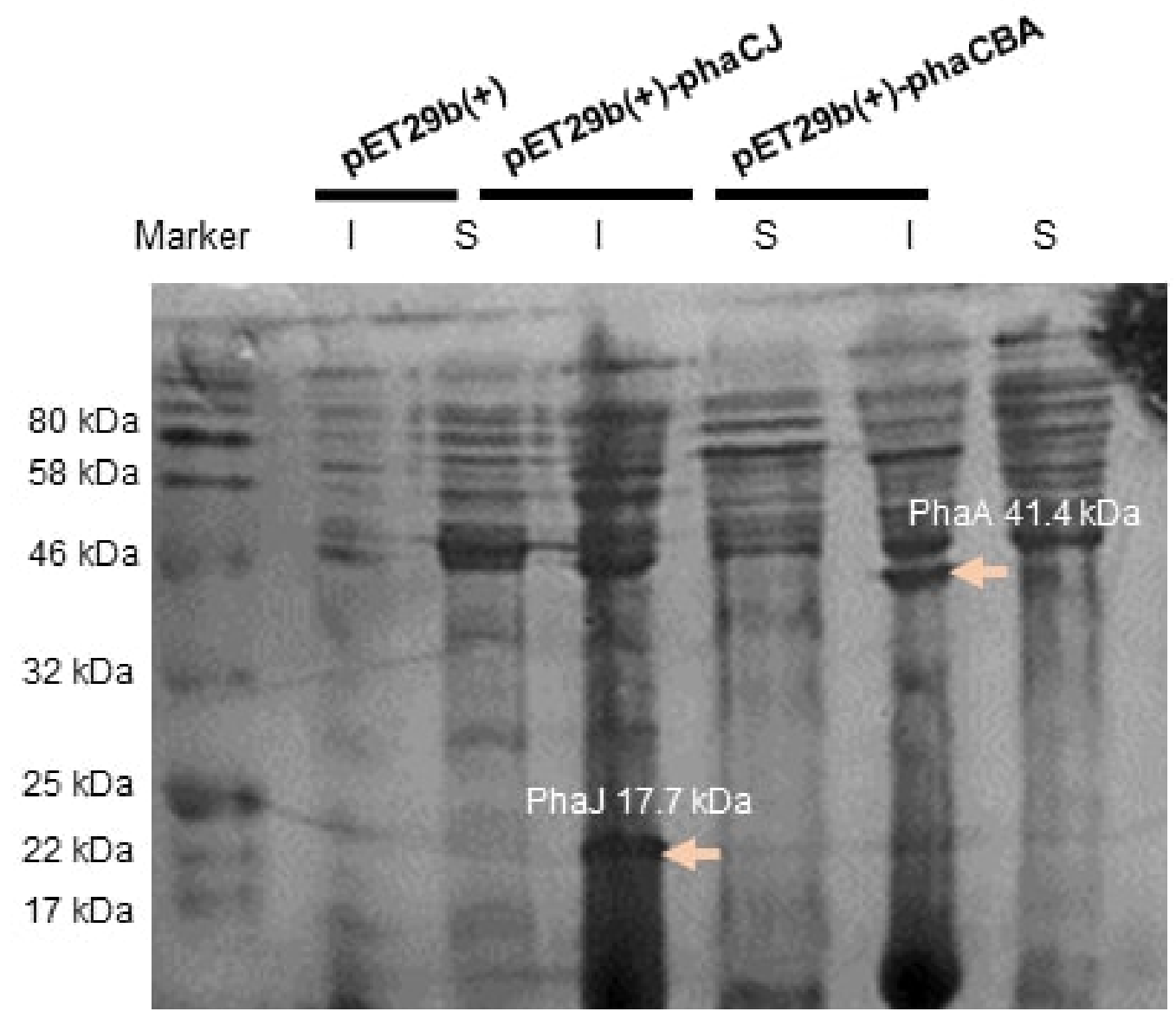

Fig 3. SDS-PAGE of E. coli containing vectors pet29b(+)-phaCJ and pet-29b(+)-phaCBA show bands of PhaJ and PhaA. SDS-PAGE of soluble (S) and insoluble (I) proteins obtained from E. coli containing pET29b(+)-phaC1J4 induced with $0.1 \mathrm{mM}$ of IPTF at $37^{\circ} \mathrm{C}$. Cells were lysed with lysozyme, sonicated, and centrifuged to divide the proteins into a soluble and insoluble fraction. The proteins were denatured after boiling. The gel was ran at $30 \mathrm{~mA}$ for 50 minutes and stained with Coomassie Blue.

To confirm the ability of $E$. coli transformed with our pET29B(+)-phaC1J4 construct to produce PHB, overnight cultures were grown for 24 hours and induced with $0.1 \mathrm{mM}$ of IPTG while supplementing them with chemical media mimicking fermented synthetic poop supernatant (FSPS). Cells were then left to culture for 16 hours. To collect the resulting $\mathrm{PHB}$, sodium hypochlorite extraction was performed. 
This work was assessed during the iGEM/PLOS Realtime Peer Review Jamboree on $23^{\text {rd }}$ February 2018 and has been revised in response to the reviewers' comments. A transcript of these comments is available with the original article as a supplementary file

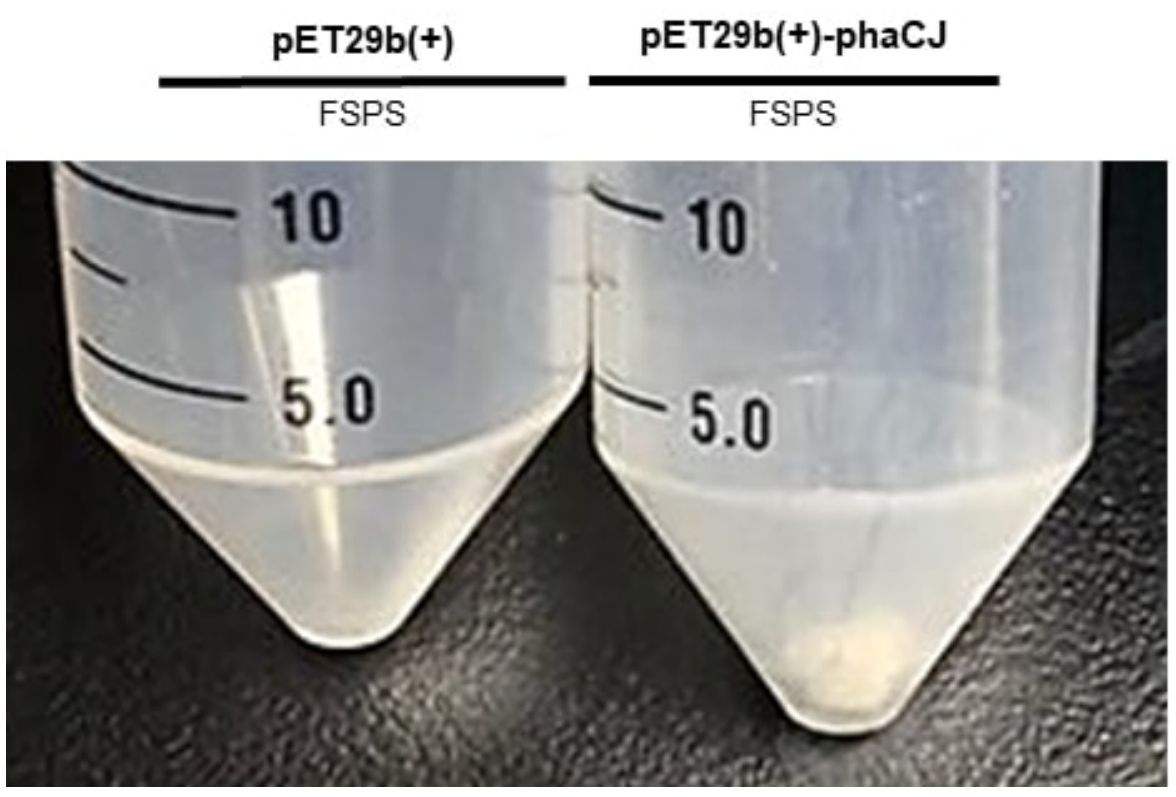

Fig 4. PHB extracted from E. coli containing pet29b(+)-phaCBA using the sodium hypochlorite extraction method. PHB extracted from phaC1J4-expressing cells cultured for 24 hours and supplemented with fermented synthetic poop supernatant (FSPS) for 16 hours. PHB was extracted using TritonX-100, sodium hypochlorite, and ethanol in a series of washes and incubation. The negative control tube contained $E$. coli transformed with the pET29B(+) vector.

To confirm the ability of $E$. coli transformed with our pET29B(+)-phaC1J4 construct to produce PHB, overnight cultures were grown for 24 hours and induced with $0.1 \mathrm{mM}$ of IPTG while supplementing them with chemical media mimicking fermented synthetic poop supernatant (FSPS). Cells were then left to culture for 16 hours. To collect the resulting $\mathrm{PHB}$, we performed sodium hypochlorite extraction.

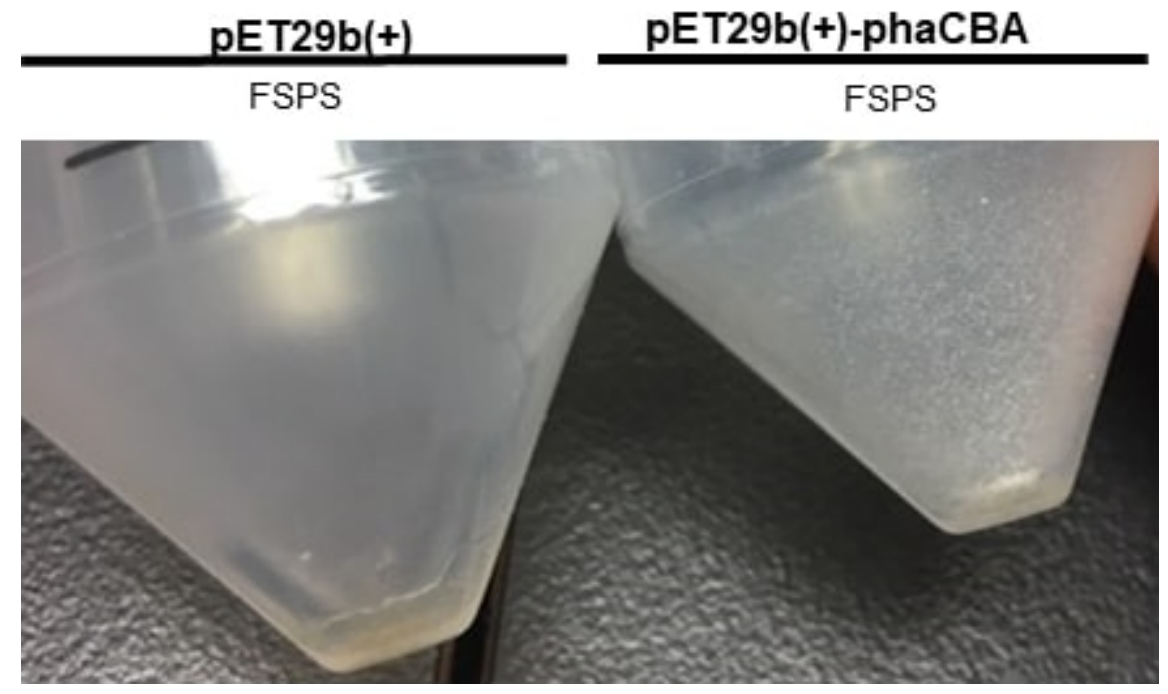

Fig 5. PHB extracted from E. coli containing pet29b(+)-phaCBA using the sodium hypochlorite extraction method. PHB extracted from phaCBA-expressing cells cultured for 24 hours and inoculated with FSPS for 16 hours. PHB was extracted using TritonX-100, sodium hypochlorite, and ethanol in a series of washes and incubation. The negative control tube contained $E$. coli transformed with the $\mathrm{pET} 29 \mathrm{~b}(+)$ vector. 


\section{PHB Characterization Using High-performance Liquid Chromatography (HPLC)}

\section{phaCBA}

The product extracted from E. coli BL21(DE3) transformed with vector containing the phaCBA operon was digested with sulfuric acid using the method in section 1. The sample was then analyzed using HPLC to detect if crotonic acid was present. The HPLC results showed that crotonic acid peak was observed. A standard curve was also generated by using known concentrations of industrially-produced PHB to determine the conversion of PHB to crotonic acid (results not shown). Hence, the amount of crotonic acid was used to determine the amount of PHB in sample. By using the predicted conversion of $80 \%$ from PHB to crotonic acid [16], the amount of crotonic acid detected was found to be $0.0282006 \mathrm{mM}$ using a dilution factor of 32 .

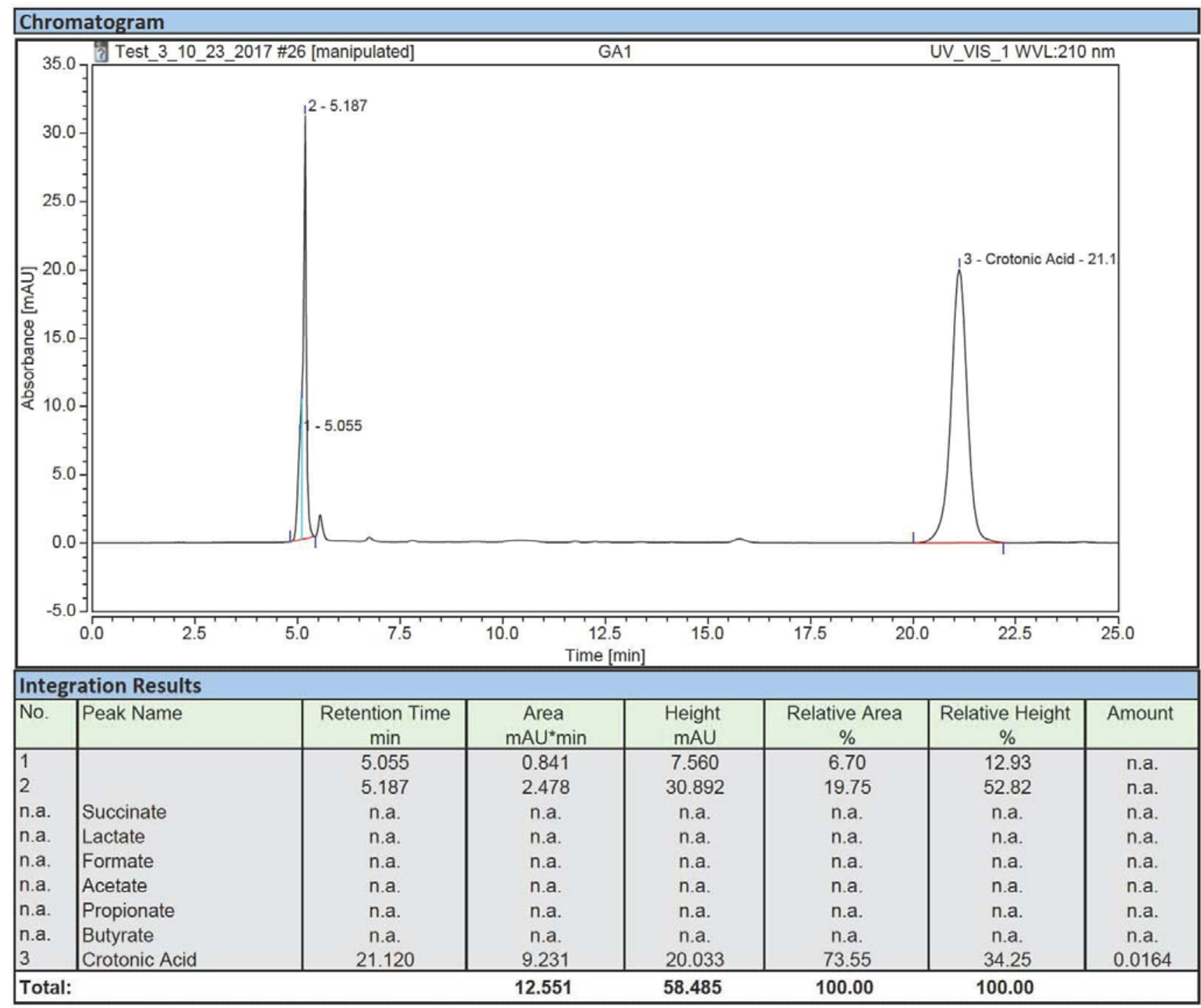

Fig 6. HPLC results of sample containing PHB digested with sulfuric acid. HPLC analysis of sample obtained from sulfuric acid digestion of product extracted from E. coli (BL21) transformed with phaCBA construct. Product was digested for 30 mins and a dilution factor of 32 was used. 
This work was assessed during the iGEM/PLOS Realtime Peer Review Jamboree on $23^{\text {rd }}$ February 2018 and has been revised in response to the reviewers' comments. A transcript of these comments is available with the original article as a supplementary file

\section{phaCJ}

Similarly, the product extracted from E. coli BL21 transformed with vector containing the phaCJ operon was digested with sulfuric acid. The sample was then analyzed using HPLC to detect if crotonic acid was present. There were two different digestion times used: 20 mins and 30 mins and low and high dilution factor for each digestion time. All samples showed a peak for crotonic acid indicating that PHB was present. However, the amount of crotonic acid detected was too low to estimate the percentage of PHB in sample.

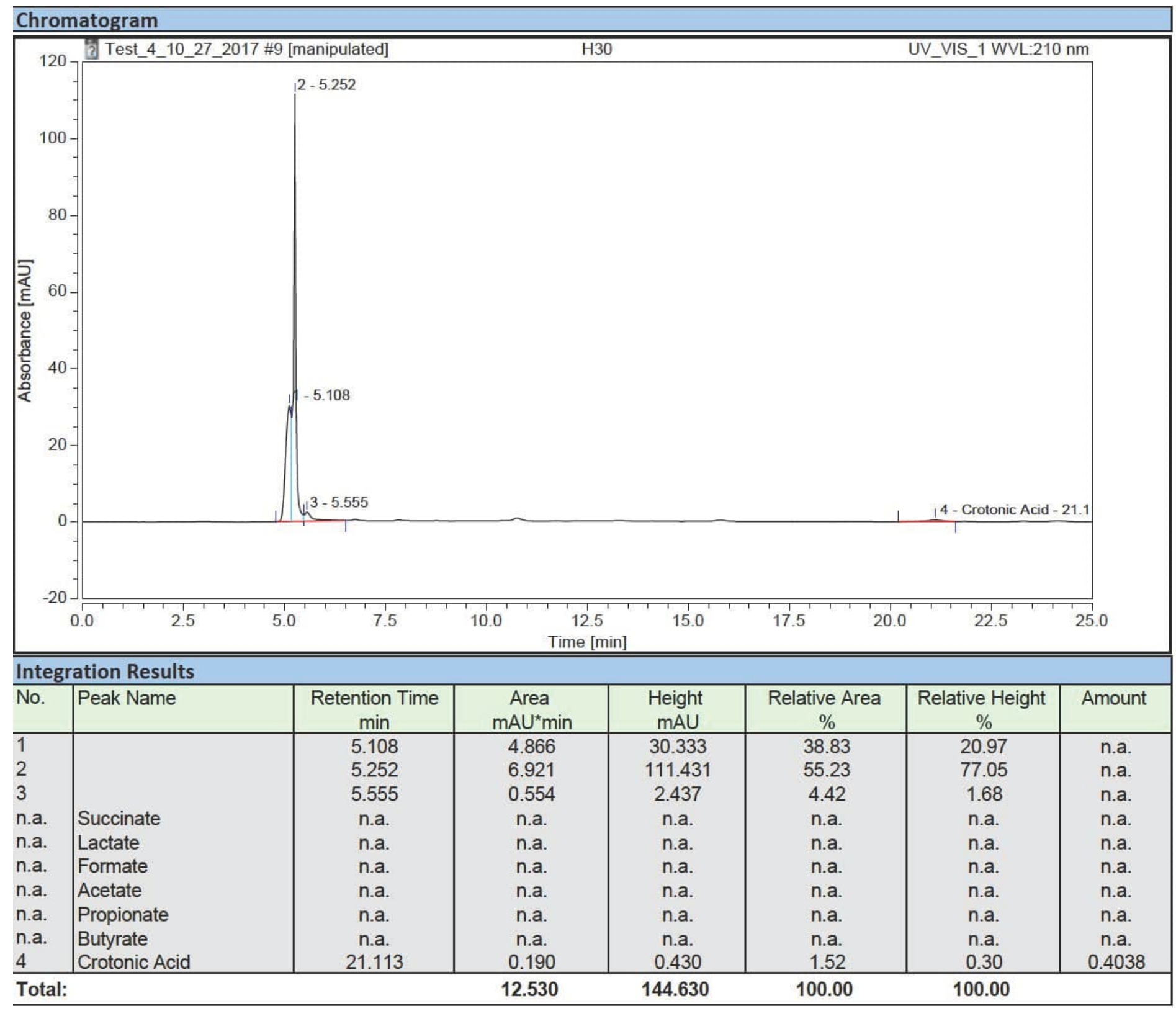

Fig 7. HPLC results of sample containing PHB digested with sulfuric acid HPLC analysis of PHB produced by $E$. coli containing pet29b(+)-phaCJ. The PHB sample was obtained from sulfuric acid digestion of product extracted from E. coli (BL21) transformed with phaCJ construct. Product was digested for 30 mins and a dilution factor of 30 was used. 


\section{Successful cloning of phasin-HlyA and phaCBA-phasin-HlyA}

The successful cloning of the pSB1C3 vector with phasin-HlyA into E. coli DH5a was demonstrated by digest confirmation with EcoRI-HF and Spel. The length of the phasin-HlyA gene is $889 \mathrm{bp}$ and the length of the pSB1C3 vector is $2.0 \mathrm{~kb}$. DNA bands of these sizes were obtained when the products of the confirmation digest were analyzed through agarose gel electrophoresis (Figure 8).

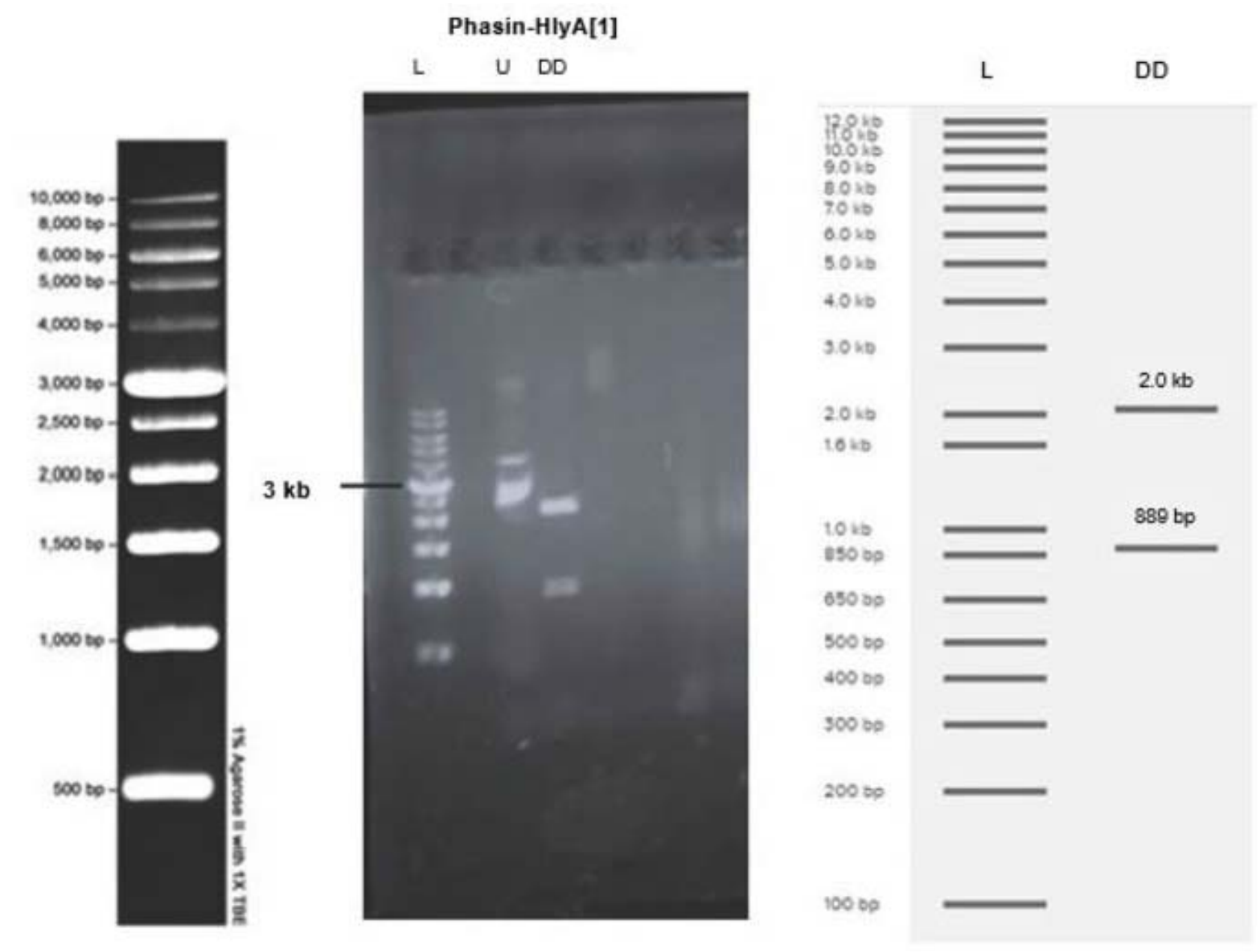

Fig 8. Screening results of DH5a transformed with phasin-HlyA in a pSB1C3 vector. (DD) Plasmid from the colony was digested with EcoRI-HF and Spel then run on a 1\% agarose gel at 100V for 30 minutes. DNA bands around $889 \mathrm{bp}$ and $2.0 \mathrm{~kb}$ are visible in lanes 3-4. (L) The molecular ladder is visible on the far left and the expected band sizes, obtained from Benchling Virtual Digest, are visible on the right. (U) Undigested plasmid was used as a control.

To create a PHB-secreting strain of $E$. coli, phaCAB was ligated into a pSB1C3 vector already containing phasinHlyA. The successful cloning of the pSB1C3 vector with phaCAB-phasin-HlyA into E. coli DH5a was demonstrated by digest confirmation with Notl-HF and Xbal. The length of the phaCAB and phasin-HlyA genes ligated together is $4.8 \mathrm{~kb}$ and the length of the pSB1C3 vector is $2.0 \mathrm{~kb}$. DNA bands of these sizes were obtained when the products of the confirmation digest were analyzed through agarose gel electrophoresis (Fig 9). Two colonies ([5] and [6]) were successfully cloned, however colony [6] was used for all secretion assays. 
This work was assessed during the iGEM/PLOS Realtime Peer Review Jamboree on $23^{\text {rd }}$ February 2018 and has been revised in response to the reviewers' comments. A transcript of these comments is available with the original article as a supplementary file

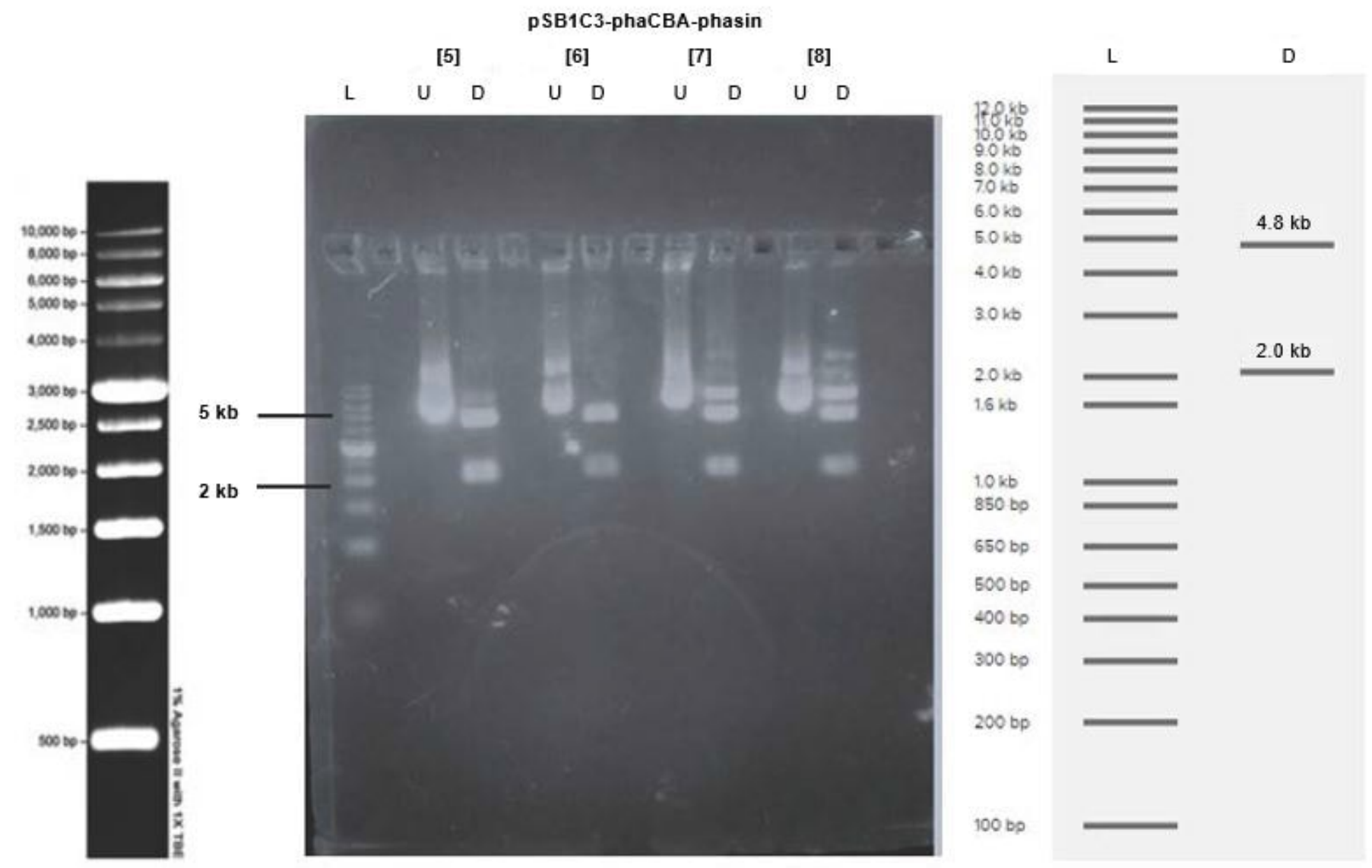

Fig 9. Screening results of DH5a transformed with pha-CAB-phasin-HlyA in a pSB1C3 vector. (D) Plasmid from the cells were digested with Notl-HF and Xbal then run on a 1\% agarose gel at $100 \mathrm{~V}$ for 30 minutes. DNA bands around $4.8 \mathrm{~kb}$ and $2.0 \mathrm{~kb}$ are visible in lanes 4 and 7, from colony [5] and [6] respectively. (L) The molecular ladder is visible on the far left and the expected band sizes, obtained from Benchling Virtual Digest, are visible on the right. (U) Undigested plasmid was used as a control.

\section{Secretion Assay}

The mass of PHB present in secreted and extracellular fractions of E.coli BL21(DE3) after the secretion assay was obtained by measuring the mass of each $50 \mathrm{~mL}$ Falcon ${ }^{\mathrm{TM}}$ tube before each trial and after the final washing and drying of the PHB step. The mass of the secreted PHB pellet was corrected by subtraction of $0.05549 \mathrm{~g}$ to account for the $\mathrm{CaCl} 2$ that was added during extraction. After 48 hours of incubation at $37^{\circ} \mathrm{C}$ there was a $114 \%$ increase in the amount of PHB secreted by cells that contain pSB1C3-phaCAB-Phasin-HlyA compared to the negative control (cells with pSB1C3-phaCAB only). However, after 24 hours of incubation at $37^{\circ} \mathrm{C}$ there was very little difference in the amount of PHB secreted by the experimental and control groups (Fig 10). 
This work was assessed during the iGEM/PLOS Realtime Peer Review Jamboree on $23^{\text {rd }}$ February 2018 and has been revised in response to the reviewers' comments. A transcript of these comments is available with the original article as a supplementary file

\subsection{5}

0.17251

0.2

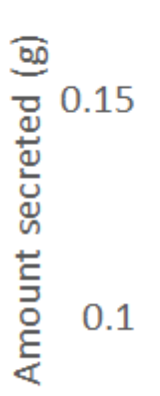

0.05

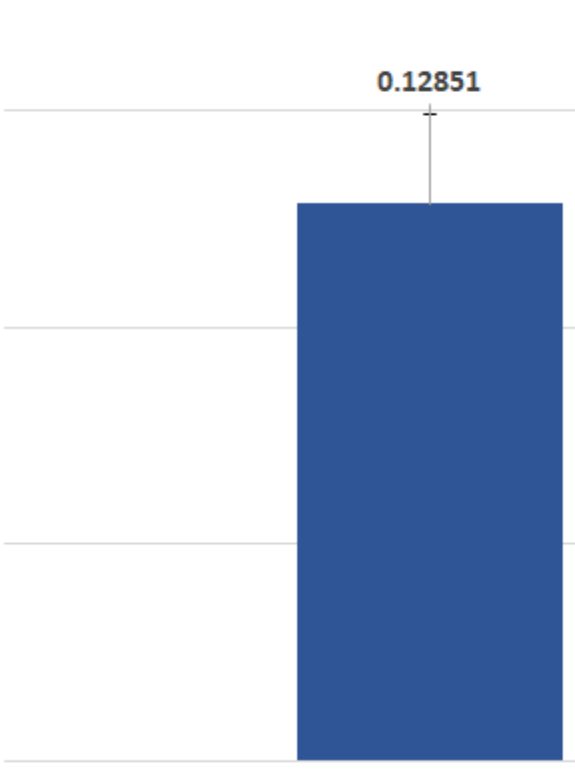

$24 \mathrm{~h}(-)$
0.12417

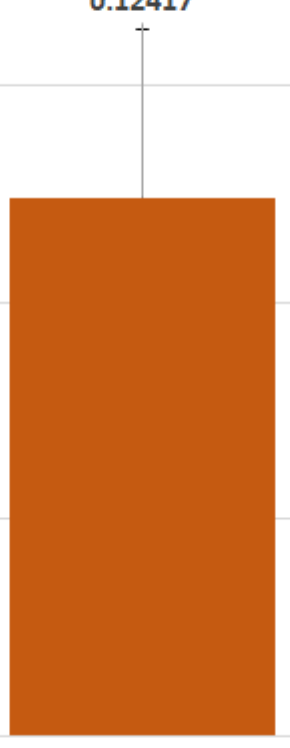

$24 \mathrm{~h}(+)$
0.08051

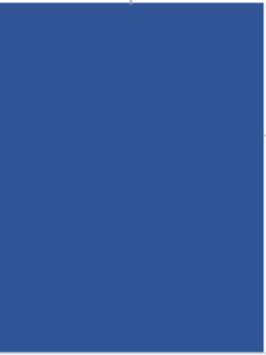

48h(-)

Fig 10. Secretion Assay Results. Amount of PHB secreted (g) after E. coli BL21(DE3) transformed with pSB1C3PhaCAB-Phasin-HlyA (pSB1C3-Phasin) or pSB1C3-PhaCAB (-) were induced then incubated in 50mL of LB (30 $\mu \mathrm{g} / \mathrm{mL}$ chloramphenicol $)+3 \%$ glucose for 24 or 48 hours. Each condition was carried out in triplicates.

\section{Process development}

\section{Effect of Temperature on VFA Fermentation}

The first VFA fermentation experiment showed higher VFA production at $37^{\circ} \mathrm{C}$ than at $22^{\circ} \mathrm{C}$ after 3 days of fermentation (Fig 11). The reported results are an average of 3 titrations performed for each sample. 
This work was assessed during the iGEM/PLOS Realtime Peer Review Jamboree on $23^{\text {rd }}$ February 2018 and has been revised in response to the reviewers' comments. A transcript of these comments is available with the original article as a supplementary file

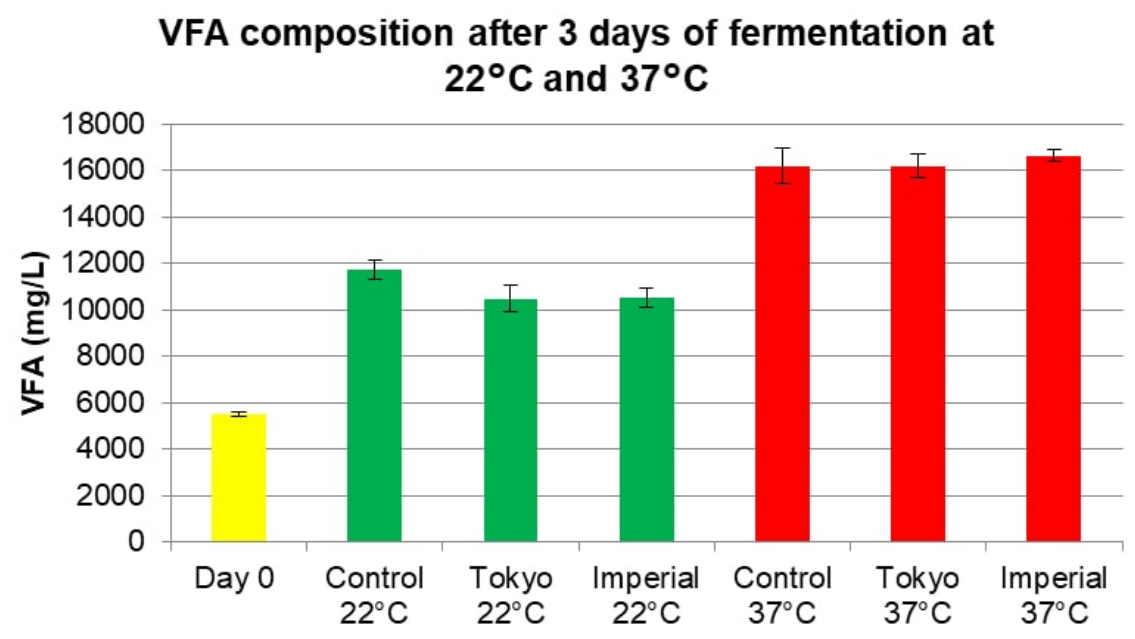

Fig 11. VFA concentrations after 3 days of fermentation at $22^{\circ} \mathbf{C}$ and $37^{\circ} \mathbf{C}$. Amount of VFA (mg/L) in the synthetic feces supernatant after a 3 day fermentation at $80 \mathrm{rpm}$ and desired temperatures with $E$. coli BL21(DE3) transformed with $\mathrm{pET} 29 \mathrm{~b}(+)$ vector without any inserts (control), PHB-producing E. coli transformed with a PHBproducing part from iGEM Imperial College 2013 ("Imperial condition”) and PHB-producing E. coli transformed with

PHB-producing part from iGEM Tokyo 2012 ("Tokyo condition”). Error bars represent standard deviation for 3 measurements.

The second VFA fermentation experiment confirmed higher VFA production at $37^{\circ} \mathrm{C}$ than at $22^{\circ} \mathrm{C}$ (Fig 12 ). In addition to a 3-day fermentation (denoted as D3), a 5-day fermentation (denoted as D5) was also introduced for this experiment. Higher VFA concentrations were observed on day 5 of fermentation. The reported results are an average of 3 titrations performed for each sample.

VFA composition after 3 and 5 days of fermentation at $22^{\circ} \mathrm{C}$ and $37^{\circ} \mathrm{C}$

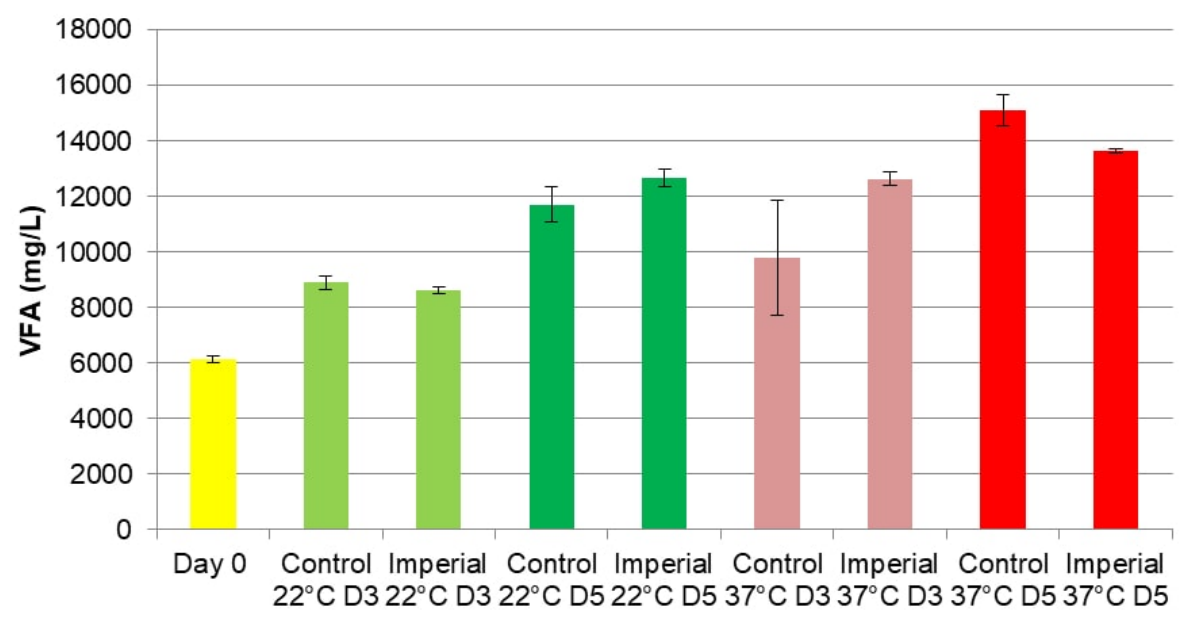

Fig 12. VFA concentrations after 3 and 5 days of fermentation at $22^{\circ} \mathbf{C}$ and $37^{\circ} \mathrm{C}$. Amount of VFA (mg/L) in the synthetic feces supernatant after 3 and 5 day fermentation at $80 \mathrm{rpm}$ and desired temperatures with $E$. coli BL21(DE3) transformed with pET29b(+) vector without any inserts (control), PHB-producing E. coli transformed with a PHB-producing part from iGEM Imperial College 2013 (“Imperial condition”). Error bars represent standard deviation for 3 measurements. 
This work was assessed during the iGEM/PLOS Realtime Peer Review Jamboree on $23^{\text {rd }}$ February 2018 and has been revised in response to the reviewers' comments. A transcript of these comments is available with the original article as a supplementary file

Next, fermented synthetic feces supernatant was sterilized and cultured with PHB-producing bacteria for 2 - 3 days at $37^{\circ} \mathrm{C}$. PHB-producing bacteria cultured in the supernatant from synthetic feces fermented at $37^{\circ} \mathrm{C}$ resulted in little to no PHB produced due to little to no bacterial growth, which is believed to be a consequence of low pH due to high concentration of VFA. Although $22^{\circ} \mathrm{C}$ resulted in lower VFA concentrations, it was selected as the preferred temperature for VFA fermentation due to optimal $\mathrm{pH}$ for bacterial growth.

\section{Equivalent System Mass Analysis}

The proposed process was divided into four main stages: collection and fermentation of feces to increase the concentration of VFA ("VFA Fermentation" step), extraction of VFA ("VFA Extraction" step), fermentation to produce PHB ("PHB Fermentation" step), and extraction of PHB from the harvest stream ("PHB Extraction"). The total calculated ESM value accounted for each step of the process as well as intermediate storage tanks and pumps (Table 5).

Table 5. Total ESM value calculated for the proposed process.

\begin{tabular}{|c|c|c|c|c|}
\hline Process Step & $\begin{array}{l}\text { Mass } \\
(\mathrm{kg})\end{array}$ & $\begin{array}{l}\text { Consumables Mass } \\
\text { (kg) }\end{array}$ & $\begin{array}{l}\text { Power } \\
\text { (kW) }\end{array}$ & $\begin{array}{l}\text { Volume } \\
\text { (m3) }\end{array}$ \\
\hline Storage tank \& pump & 56 & 0 & 1.8 & 0.05 \\
\hline VFA Fermentation & 20 & 0 & 0.02 & 0.01 \\
\hline VFA Extraction & 25 & 0 & 2.2 & 0.01 \\
\hline $\begin{array}{l}\text { PHB Fermentation - } \\
\text { bioreactor }\end{array}$ & 15 & 0 & 0.4 & 0.12 \\
\hline $\begin{array}{l}\text { PHB Fermentation - self- } \\
\text { cleaning filter }\end{array}$ & 16 & 0 & 0.19 & 0.03 \\
\hline PHB Extraction & 42 & 0 & 0.62 & 1 \\
\hline Total & 174 & 0 & 5.2 & 1.2 \\
\hline ESM (kg) & 900 & & & \\
\hline
\end{tabular}

The total ESM value for our process is lower than some of the systems currently used on the International Space Station. For example, the Water Processor (WP) unit on the International Space Station has an ESM value of about $5,000 \mathrm{~kg}$ [22]. After comparing our process to existing life support systems on the International Space Station, we concluded that our process is feasible.

\section{Chemical coagulation of PHB}

We demonstrated in the lab that adding calcium chloride and then centrifuging increased the amount of PHB removed from a suspension in distilled water. We carried out centrifugation at various speeds and then collected absorbance data for the samples to estimate relative quantity of PHB remaining in suspension. The lower the absorbance reading, the higher the amount of PHB that was removed. Absorbance readings and the standard deviations of the various conditions tested are shown in the Fig 13. 


\section{Extraction Methods}

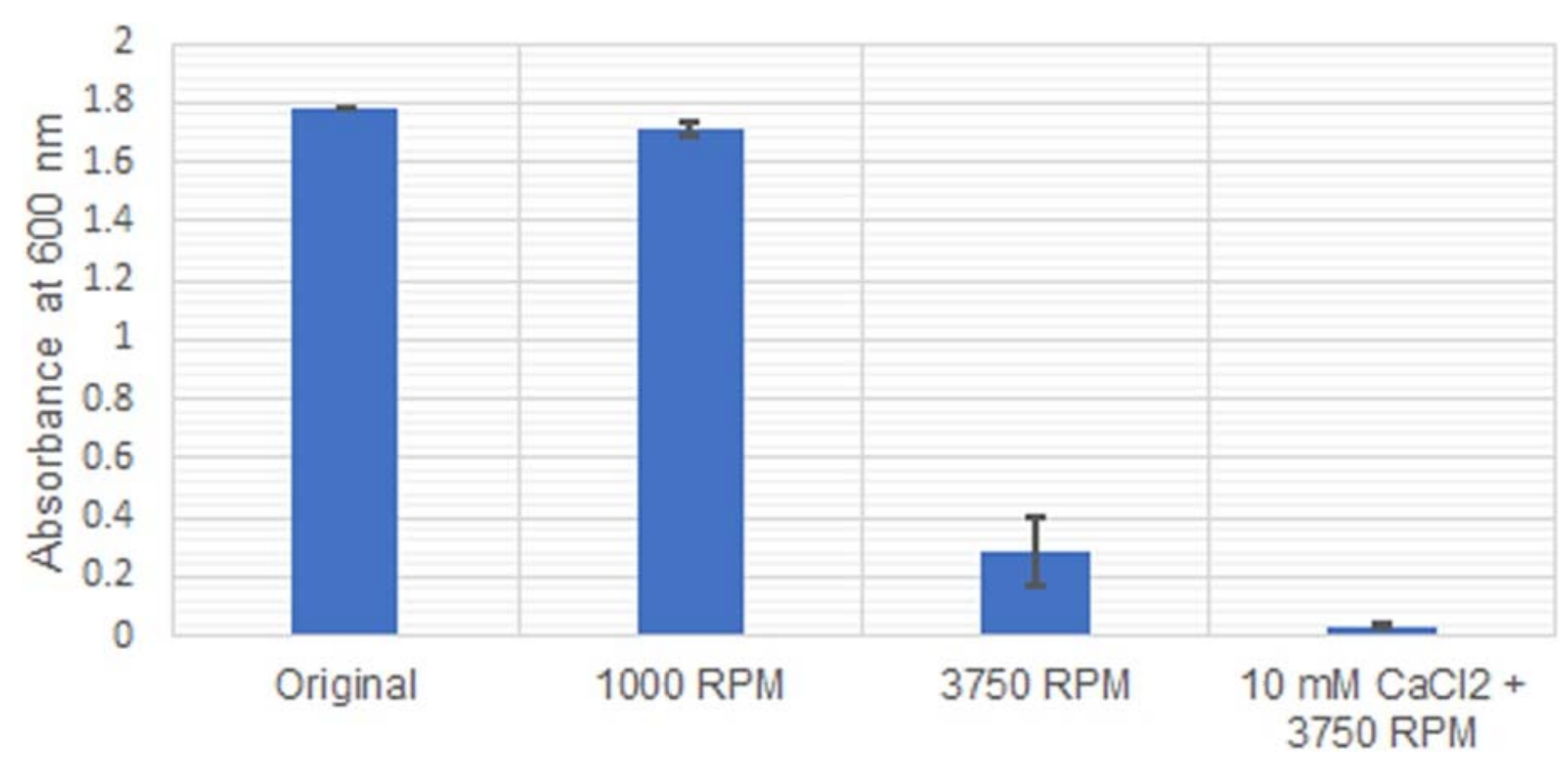

Conditions Tested

Fig 13. Absorbance readings and their standard deviations for the various extraction methods tested.

As expected, centrifugation at higher RPM and addition of calcium chloride resulted in greater removal of PHB from suspension.

\section{Electrocoagulation of PHB}

From our preliminary experiments with just PHB particles in suspension in distilled water, we were able to demonstrate that PHB particles could settle out via electrocoagulation. A layer of PHB was observed at the bottom of the electrocoagulation cell after it was run for 3 hours. However, a few hours after the cell was shut down, a thin layer of brown powder settled on top of the white layer of PHB. We hypothesized that this was probably iron(III) hydroxide, formed from the excess iron ions released by the anode that did not bind to the PHB.

From our experiments with synthetic feces supernatant, we found that a layer of brown sludge settled at the bottom each time. Even with a 1:1 mixture of PHB suspension and synthetic feces supernatant there was no discernable layer of PHB within the sludge. A sample of the sludge was washed with dilute acid to remove the metal salts that might have been present. However, we were still unable to separate PHB from the sludge.

The electrocoagulation experiments led us to conclude that while it was possible to settle out PHB using electrocoagulation, this method was not suitable for our media which contained a number of salts that interfered with the coagulation process and caused the formation of sludge. 


\section{Discussion}

\section{Synthesis}

\section{Construct}

phaCJ and phaCBA were successfully cloned into pET29b(+) vectors as shown in the results (Fig 1., Fig 2.) The gel electrophoresis bands of the digested vectors were compared with the expected bands generated from Benchling. Fig1. showed 2 distinct bands for each lane that contained plasmids extracted from various colonies of $E$. coli transformed with the pET29b(+)-phaCJ plasmid. The band sizes were approximately $5.4 \mathrm{~kb}$ and $2.2 \mathrm{~kb}$, corresponding to the predicted bands. Fig 2. verified the successful cloning of pET29b(+)-phaCBA. The expected bands were seen at $5.3 \mathrm{~kb}, 2.8 \mathrm{~kb}$, and $1.0 \mathrm{~kb}$. The expected bands image showed an additional band $16 \mathrm{bp}$ in size, but this band was not seen in our experimental electrophoresis gel. This is likely because $16 \mathrm{bp}$ is a minute size for the DNA examined. Therefore, the DNA of this size may have run off the gel before the current was stopped. However, another gel would have to be run at a shorter time to detect the presence of this $16 \mathrm{bp}$ DNA band. Overall, the gel electrophoresis provided sufficient evidence of the successful ligation of the two genes.

\section{Enzyme Production}

The SDS-PAGE results in Fig 3. showed clear bands for PhaJ (17.7 kDa) and PhaA (41.4 kDa), indicating the expression of the genes from the plasmids. These protein bands were in the insoluble protein fraction. This finding contrast past literature that has found PhaJ in the soluble protein fraction for SDS-PAGE [23]. However, McCool and Cannon found that polyhydroxyalkanoates tend to localize to inclusion bodies, providing an explanation to why these proteins are seen in the insoluble protein fraction [24].

phaJ was part of the pet29b(+)-phaCJ plasmid and the phaA was part of the pet29b(+)-phaCBA plasmid. The identification of other bands for the corresponding proteins in the same expression plasmid was difficult to identify due to lack of clarity and the closeness of the bands in the regions of interest. Although it is reasonable to assume that the other proteins were also produced alongside the proteins detected in the SDS-PAGE because the genes encoding for the rest of the proteins are within the same plasmid and open reading frame, further validation will be needed to verify their expression. Fig 3. did not show PhaC $(63.4 \mathrm{kDa})$ from pet29b(+)-phaCJ, PhaC $(65.1 \mathrm{kDa})$ and PhaB (27.2 kDa) from pet29b(+)-phaCBA. However, because these genes all have their own $6 x H i s$ tag, protein purification may be performed to eliminate excess bands to isolate the proteins of interest for a future SDS-PAGE. In addition, a Western Blot can be used to further confirm the protein production.

\section{PHB synthesis}

The results showed that PHB was produced by cells containing the phaCBA operon and the phaC1J4 biobrick, as evidenced by the presence of white powder after a sodium hypochlorite PHB extraction from cells containing both the phaCBA and phaCJ4 constructs (Fig 6). As discussed below, this white powder was confirmed to be PHB by HPLC analysis. However, the mass of white powder produced by $10 \mathrm{~mL}$ of $E$. coli cultures was negligible, and while we could visually confirm PHB production, there was no way to accurately gauge the mass of PHB produced during the lab-scale tests which were conducted. Future tests would need to use more sensitive methods of PHB quantification or work with larger volumes of $E$. coli cultures. 


\section{HPLC Analysis - phaCBA and phaC1J4}

Samples obtained through chemical extraction of E. coli transformed with our genetic constructs were digested with sulfuric acid, and the resulting digest was analyzed using HPLC. HPLC analysis of the sample from E. coli transformed with $\mathrm{pET} 29 \mathrm{~b}$ plasmid containing phaCBA operon showed that $0.0282006 \mathrm{mM}$ of crotonic acid was produced when a dilution factor of 32 was used and product digested for 30 mins. HPLC analysis of the digested product obtained from sulfuric acid digestion of sample when $E$. coli transformed with pET29b plasmid containing phaCJ operon was used also indicated that crotonic acid was present. Hence, the samples obtained using the two genetic constructs contained PHB. However, the amount of PHB in the sample could not be determined because the amount of crotonic acid detected was too low. The low concentration of crotonic acid could have been due to a number of reasons. Long duration of digestion of sample could have resulted in degradation of crotonic acid. In order to avoid degradation, an optimal digestion time needs to be selected by conducting HPLC with various digestion times. The method of PHB extraction could also be optimized to minimize the loss of PHB. Nonetheless, HPLC was successful in confirming production of PHB, but due to a number of limitations the amount of PHB in the samples could not be determined.

\section{Secretion}

The results show that 48 hours after induction initiation there was a 114\% increase in the amount of PHB secreted by cells that contain phaCAB-phasin-HlyA compared to control cells, which contain pSB1C3-phaCAB only. However, there is very little difference between the two groups at 24 hours (Fig 10). Therefore it can be inferred that PHB secretion with phasin-HlyA occurred only after at least 24 hours the induction of cultures with IPTG. These results demonstrate that the type I secretion mechanism in E. coli can successfully secrete PHB when coupled with the phasin molecule from $R$. eutropha. The production of "secreted" PHB in control cells may not be PHB that was actually secreted, but instead it may be PHB that was released into the media as a result of cell death and lysis, which is a normal part of the E. coli life-cycle. Rahman et. al obtained similar results in their 2013 study on the secretion of PHB in E. coli, in which an initiation of PHB secretion between 24 and 48 hours after induction was observed [9].

\section{Process Development}

\section{Process Overview}

The proposed process that converts astronauts' fecal matter to a bioplastic that can be used to 3D print tool on Mars consists of four major steps (Fig 14). In the first step, astronauts' feces are collected into a storage tank using a vacuum toilet. Feces are then transferred to a fermenter for 3 days, where natural gut flora produces VFA. In the second step, the liquid containing VFA along with other nutrients found in human feces are separated from solid particles using centrifugation followed by a self-cleaning filter. The obtained liquid is passed to another storage tank. In the third step, the supernatant containing VFA is added to a fermenter, which is then inoculated with PHBproducing and PHB-secreting E. coli. In the fourth and last step, PHB secreted by bacteria is extracted from the liquid harvest stream using dissolved air flotation. After drying, the PHB can be used in a Selective Laser Sintering (SLS) 3D printer without additional processing [14]. Each step of the process is further explained in subsequent sections. 
This work was assessed during the iGEM/PLOS Realtime Peer Review Jamboree on $23^{\text {rd }}$ February 2018 and has been revised in response to the reviewers' comments. A transcript of these comments is available with the original article as a supplementary file

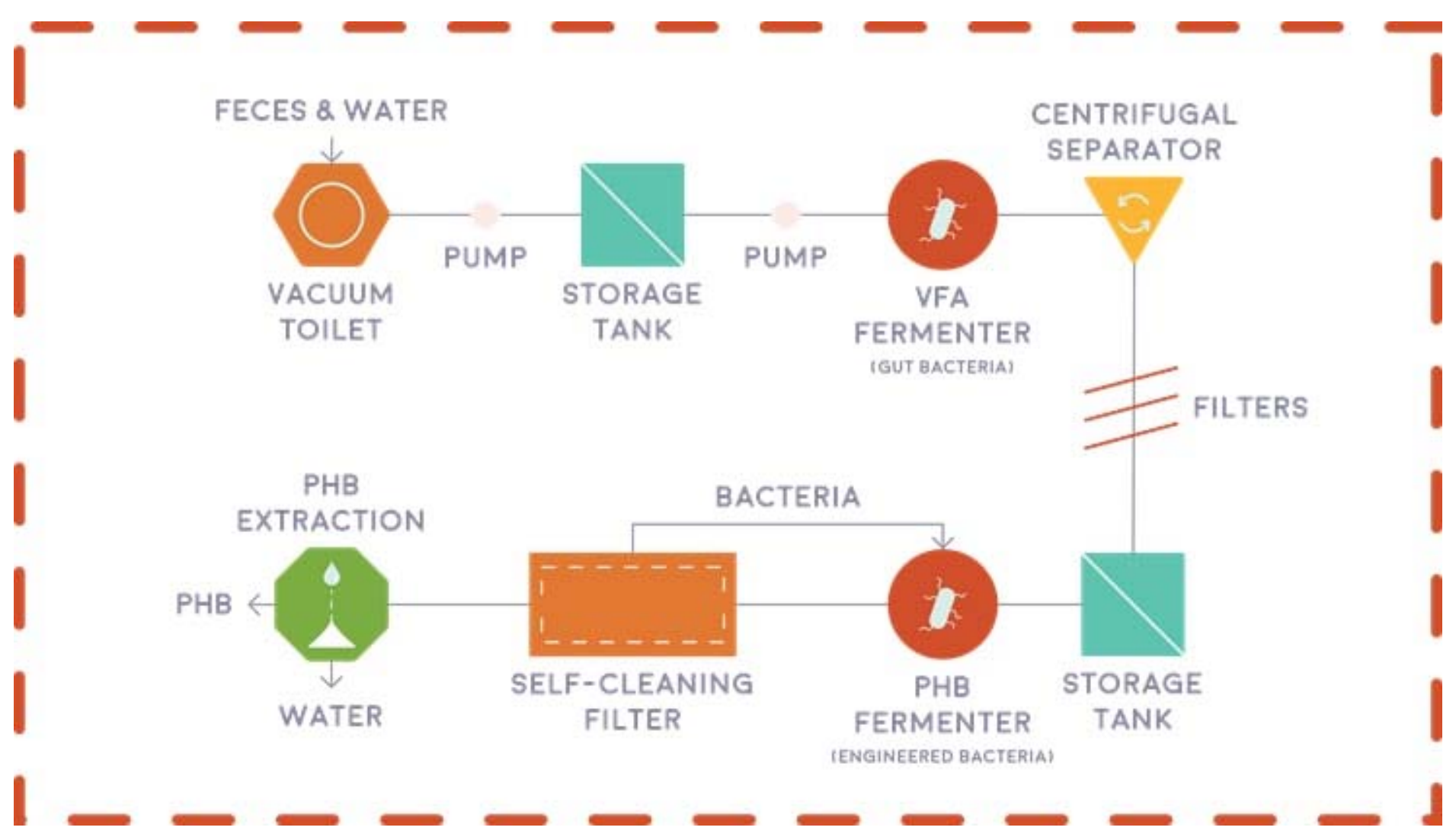

Fig 14. Overview of the proposed process that starts with astronauts' feces and end with a bioplastic product that can be used to 3D print tool for astronauts.

\section{VFA Fermentation}

In this step, astronauts' feces are collected into a storage tank using a vacuum toilet. Although the vacuum toilet requires about $0.5 \mathrm{~L}$ of water, which is a valuable resource on Mars, per flush, the used water can be recovered at the end of the process using a water processing unit currently used on the International Space Station. The storage tank was designed based on NASA requirements for a fecal collection system, which must be able to collect an average of $150 \mathrm{~mL}$ of fecal matter per defecation for 2 defecations per crew member per day [21]. Additionally, the system must be designed to contain $1.5 \mathrm{~L}$ of diarrhea discharge. To meet the requirements of our process, the storage tank should also be able to hold 3 days' worth of fecal matter. Taking these requirements into account with an additional $0.5 \mathrm{~L}$ of water entering the storage tank per flush and a crew of 6 astronauts, the volume of the storage tank was found to be $15 \mathrm{~L}$. After the storage tank, feces are fermented in another vessel with naturally occuring bacteria for 3 days at $22^{\circ} \mathrm{C}$ (room temperature) to increase the concentration of VFA. The fermenter will use an agitator to prevent the settling of solids and bacteria during fermentation. Temperature, $\mathrm{pH}$, and dissolved oxygen will not be controlled.

\section{VFA Extraction}

In this step, the liquid is recovered from feces and separated from solid particles to obtain a sterile, VFA-rich stream for the PHB fermentation step. Large solids are removed using centrifugation and smaller solids along with bacteria are then removed using a self-cleaning filter.

Alternative designs considered for this step included torrefaction and a screw press dewatering system. Torrefaction is defined as thermochemical treatment of biomass in the absence of oxygen and can recover both natural and 
This work was assessed during the iGEM/PLOS Realtime Peer Review Jamboree on $23^{\text {rd }}$ February 2018 and has been revised in response to the reviewers' comments. A transcript of these comments is available with the original article as a supplementary file

pyrolytic water. While the torrefaction process was a better alternative based on ESM, the liquid product of torrefaction containing water and VFA was too acidic and lacked required nutrients to support bacterial growth. Centrifugation followed by filtration recovered sufficient amounts of water and had a lower ESM value than a screw press dewatering system. However, we propose torrefaction treatment for the sludge remaining after centrifugation. Torrefaction treatment would recover additional water and produce char, which can be used as a building material, radiation shielding or as a food substrate.

\section{PHB Fermentation}

In this step, VFA-rich stream is fermented with PHB-producing E. coli in a continuous process. The process occurs in a $5-\mathrm{L}$ stirred-tank bioreactor at $37^{\circ} \mathrm{C}$ under anaerobic conditions. Continuous fermentation is achieved using a self-cleaning filter with 0.2 um pores, which separates bacteria from the liquid harvest stream and recycles bacteria back into the bioreactor (Fig 15). The resulting bacteria-free liquid harvest stream contains PHB secreted by bacteria, dissolved salts, and unused volatile fatty acids.

To achieve a continuous fermentation system, alternative designs considered for this step included various configurations of membrane bioreactors. However, the major disadvantage of membrane bioreactors is fouling, resulting in high crew time requirements and transportation costs due to consumables.

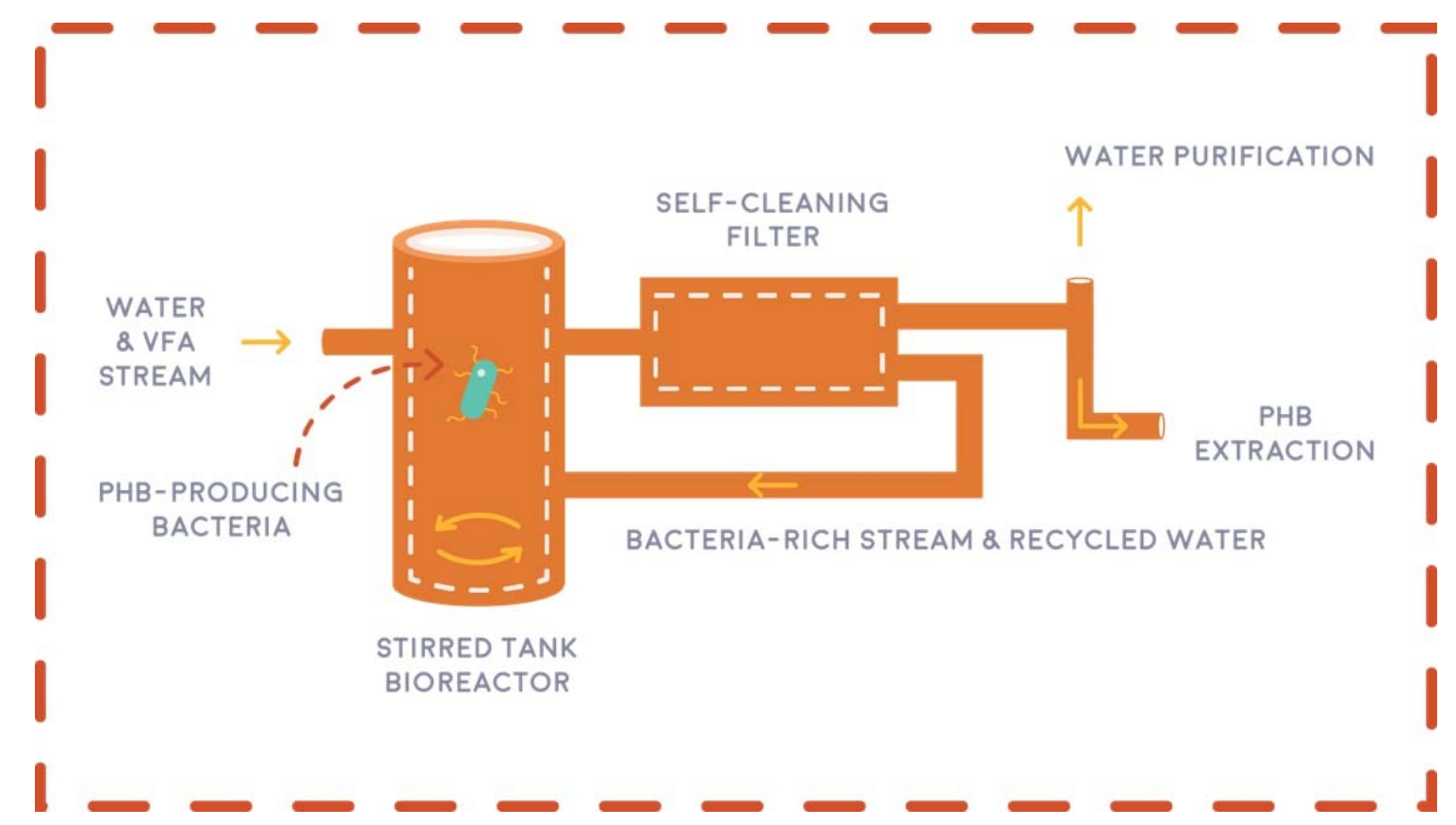

Fig 15. Overview of the proposed PHB fermentation process, where PHB is continuously produced using engineered bacteria.

\section{PHB Extraction}

Extraction of PHB nanoparticles from the liquid harvest stream is achieved using dissolved air flotation (DAF) (Fig 16). In DAF, air saturated water is injected into the vessel containing PHB particles suspended in liquid. The resulting air bubbles capture PHB nanoparticles and float up, resulting in accumulation of PHB at the top of the liquid. This layer of PHB can then be removed and dried to obtain the final product. 
This work was assessed during the iGEM/PLOS Realtime Peer Review Jamboree on $23^{\text {rd }}$ February 2018 and has been revised in response to the reviewers' comments. A transcript of these comments is available with the original article as a supplementary file

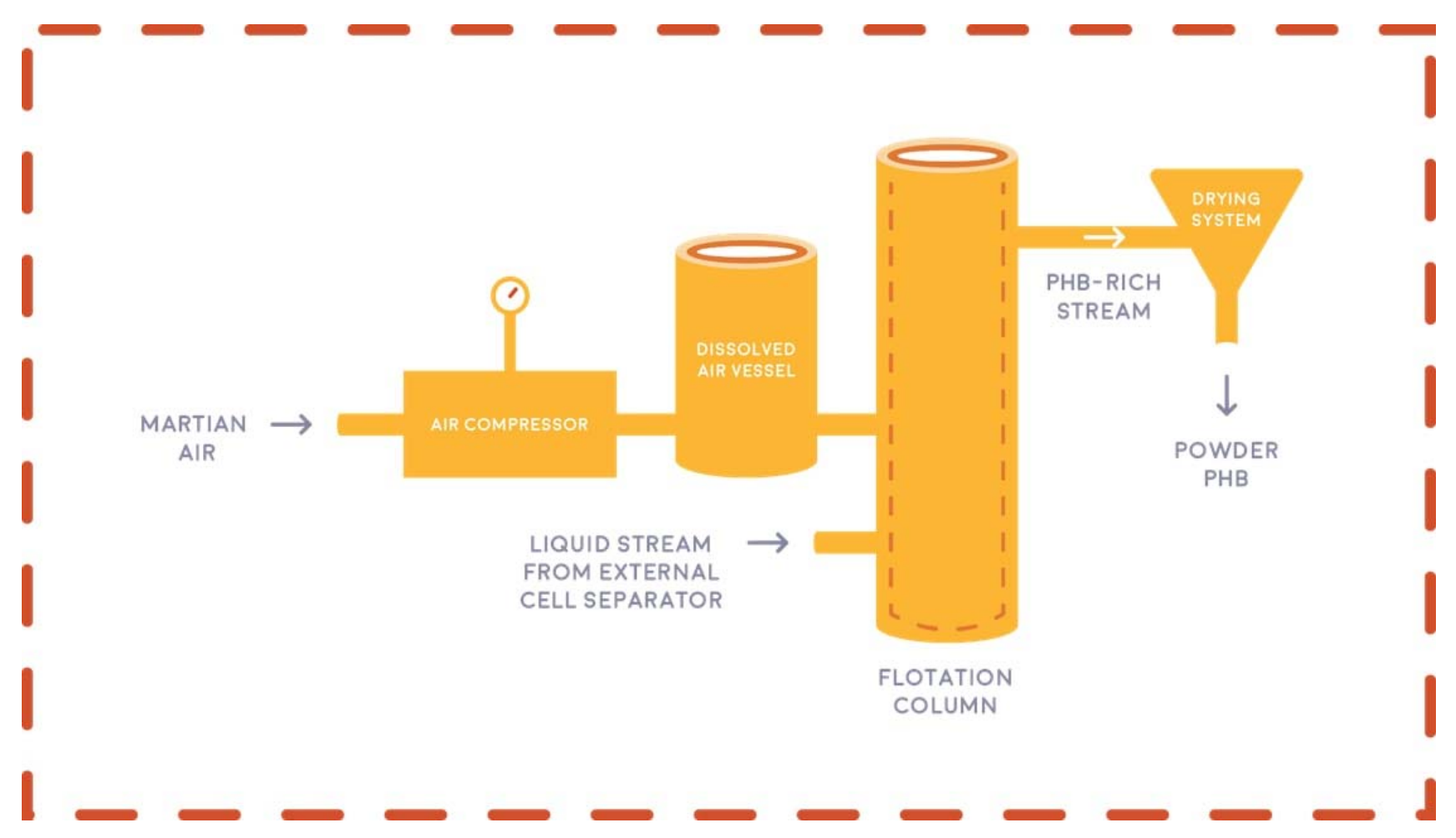

Fig 16. Overview of the proposed PHB extraction method using dissolved air flotation.

Alternative designs considered for this step included chemical coagulation and electrocoagulation. While chemical coagulation would require shipment of chemicals to Mars, electrocoagulation resulted in accumulation of sludge along with PHB during laboratory experiments. Dissolved Air Flotation was therefore selected as the preferred design option.

\section{Applied Design}

When developing the physical process on Mars that starts with feces and ends with the bioplastic product, we identified design requirements based on advice from experts. Then, we iterated the design of our process based on feedback from space industry professionals including Canadian astronauts Dr. Robert Thirsk and Col. Chris Hadfield, Dr. Matthew Bamsey (Chief Systems Engineer at the German Aerospace Center), and Dr. Pascal Lee (Principal Investigator of the Haughton-Mars Project at NASA Ames Research Center and a co-founder of the Mars Institute). Furthermore, we used ESM analysis, a method recommended by NASA specifically for advanced life support systems, to evaluate the feasibility of our process. Additionally, we investigated applications for solid human waste that NASA is considering and whether the byproducts of our process have any useful applications. These considerations would allow us to integrate our process with NASA's systems. Lastly, we considered the safety of our design as it was identified as one of the most important criteria based on interviews with experts.

\section{Conclusion \& Future Directions}

The key aims of this project were to optimize PHB production in E. coli, to optimize PHB secretion from E. coli to the surrounding media, and to design a start-to-finish process for PHB production from human waste on a potential future Mars base. Further testing needs to be done to definitively claim that the first two aims were accomplished. The third aim (the development of the PHB production/ waste management process for Mars bases) has been 
This work was assessed during the iGEM/PLOS Realtime Peer Review Jamboree on $23^{\text {rd }}$ February 2018 and has been revised in response to the reviewers' comments. A transcript of these comments is available with the original article as a supplementary file

partially achieved, with all components of the process accounted for and several components confirmed to work at the lab scale.

The dissolved air flotation aspect of the PHB production process will be further developed as a part of the Canadian Reduced Gravity Experiment Challenge (Can-RGX), which will take place in the summer of 2018. As a part of Can$\mathrm{RGX}$, the dissolved air flotation aspect of our project will be optimized for microgravity in order for PHB production to occur en route to Mars (as well as on the Martian surface).

\section{Acknowledgements}

Dr. Mayi Arcellana-Panlilio - the primary supervisor of iGEM Calgary 2017. Without her guidance and support, the project would not have been realized.

Rachelle Varga - a Teaching Assistant for iGEM Calgary 2017. She assisted with laboratory discussions, as well as supervision, and provided the team with applicable protocols.

David Feehan - a Teaching Assistant for iGEM Calgary 2017. He provided the team with supervision and advice on laboratory techniques.

Dr. Richard Moore - provided the transformation protocol, which which was used to transform the E. coli BL21 (DE3) and $E$. coli DH5a used in the experiments.

Dr. Elke Monika Lohmeier-Vogel - gave feedback on the progress of the project and helped research the biochemical pathways of PHB production with the team.

Dr. Craig Jenne - advised the team on the safety aspects of the project on the users and the surrounding environment on Mars.

Dr. Fabiola Aparicio-Ting - guided us on the ethical and societal considerations of the project.

Brad Prince - Provided the cost analysis of PHB production using synthetic biology.

Dr. Nashaat Nassar - helped with process development and suggested the use of electrical coagulation for PHB extraction.

Anirban Chakraborty and Jayne Rattray - trained the team on using the HPLC and helped set up runs and methods, as well as troubleshooting, for VFA and PHB quantification. 
This work was assessed during the iGEM/PLOS Realtime Peer Review Jamboree on $23^{\text {rd }}$ February 2018 and has been revised in response to the reviewers' comments. A transcript of these comments is available with the original article as a supplementary file

\section{References}

1. Hsu., J. (2011). Total Cost of NASA's Space Shuttle Program: Nearly $\$ 200$ Billion. Space.com (Magazine). Retrieved September 17, 2017, from https://www.space.com/11358-nasa-space-shuttle-program-cost-30years.html

2. Albuquerque, M.G.E., Martino, V., Pollet, E., Avérous, L. \& Reis, M.A.M. (2011). Mixed culture polyhydroxyalkanoate (PHA) production from volatile fatty acid (VFA)-rich streams: Effect of substrate composition and feeding regime on PHA productivity, composition and properties. J. Biotechnol., 151(1): 66-76

3. Rose, C., Parker, A., Jefferson, B. \& Cartmell, E. (2015). The characterization of feces and urine: a review of the literature to informed advanced treatment technology. Critical Reviews in Environmental Science Technology. 45: $1827-1879$

4. Hiroe A, Tsuge K, Nomura CT, Itaya M, Tsuge T. Rearrangement of gene order in the phaCAB operon leads to effective production of ultrahigh-molecular-weight poly[(R)-3-hydroxybutyrate] in genetically engineered Escherichia coli. Appl. Environ. Microbiol. 2012;78: 3177-84.

5. Black, P.N. \& DiRusso, C.C. (1994). Molecular and biochemical analyses of fatty acid transport, metabolism, and gene regulation in Escherichia coli. Biochimica et Biophysica Acta. 1210: 123-145

6. Davis, R., Anilkumar, P.K., Chandrashekar, A. \& Shamala, T.R. Biosynthesis of polyhydroxyalkanoates copolymer in E. coli using genes from Pseudomonas and Bacillus. Antonie Van Leeuwenhoek. 2018;94: 207-216

7. Tsuge T, Taguchi K, Taguchi S, Doi Y. Molecular characterization and properties of (R) - specific enoyl-CoA hydratases from Pseudomonas aeruginosa: metabolic tools for synthesis of polyhydroxyalkanoates via fatty acid B-oxidation. Int. J. Biol. Macromolec. 2003;31(4-5): 195-205.

8. Lu, X., Zhang, J., Wu, Q. \& Chen, G.Q. (2003) Enhanced production of poly(3-hydroxybutyrate-co-3hydroxyhexanoate) via manipulating the fatty acid beta-oxidation pathway in E. coli. FEMS Microbiol Lett. 221: 97-101

9. Rahman, A., Linton, E., Hatch, A., Sims, R., Miller, C. Secretion of polyhydroxybutyrate in Escherichia coli using a synthetic biological engineering approach. J Biol Eng. 2013; 7(1): 24.

10. Hahn, K.S., Chang, Y.K, Kim, B.S. \& Chang, H.N. Optimization of microbial poly(3-hydroxybutyrate) recovery using dispersions of sodium hypochlorite solution and chloroform. Biotechnol. Bioeng. 1994; 44: 256-261.

11. George, H.J., Watson, R.J., Harbrecht, D.F. \& DeLorbe, W.J. A bacteriophage lambda cl857 cassette controls lambda pL expression vectors at physiologic temperatures. Nat. Biotechnol. 1987; 5: 600-603.

12. Thomas, S., Holland, I.B. \& Schmitt, L. The type I secretion pathway: the hemolysin system and beyond. Biochimica et Biophysica Acta. 2014; 18(43): 1629-1641.

13. Maehara, A., Ueda, S., Nakano, H. \& Yamane, T. Analyses of a polyhydroxyalkanoic acid granule-associated 16-kilodalton protein and its putative regulator in the pha locus of paracoccus denitrificans. J. Bacteriol. 1999; 181: 2914-2921.

14. Pereira TF, Oliveira MF, Maia IA, Silva JVL, Costa MF, Thiré RMSM. 3D Printing of Poly(3-hydroxybutyrate) Porous Structures Using Selective Laser Sintering. Macromol Symp [Internet]. 2012;319(1):64-73.

15. Maloy, S. Codon usage in E. coli [internet]. 2002. Available from:

http://www.sci.sdsu.edu/ smaloy/MicrobialGenetics/topics/in-vitro-genetics/codon-usage.html 
This work was assessed during the iGEM/PLOS Realtime Peer Review Jamboree on $23^{\text {rd }}$ February 2018 and has been revised in response to the reviewers' comments. A transcript of these comments is available with the original article as a supplementary file

16. Karr, D. B., Waters, J. K., \& Emerich, D. W. Analysis of poly- $\beta$-hydroxybutyrate in Rhizobium japonicum bacteroids by ion-exclusion high-pressure liquid chromatography and UV detection. Appl. Environ. Microbiol.1983;46(6):1339-44.

17. Hackaday.io. Make Water - Collaborative Water Purification [Internet]. 2017. Available from: https://hackaday.io/project/20812-make-water-collaborative-water-purification\#menu-description

18. Wignarajah K, Litwiller E, Fisher J, Hogan J. Simulated Human Feces for Testing Human Waste Processing Technologies in Space Systems. SAE Transactions, J Aerosp. 2006;115(1).

19. Anderson GK, Yang G. Determination of bicarbonate and total volatile acid concentration in anaerobic digesters using a simple titration. Water Environ Res. 1992;64(1):53-9.

20. Levri JA, Drysdale AE, Ewert MK, Fisher JW, Hanford AJ, et al. Advanced Life Support Equivalent System Mass Guidelines Document. NASA Tech Rep. 2003;(September).

21. Anderson MS, Ewert MK, Keener JF, Wagner SA. Life Support Baseline Values and Assumptions Document. Nasa/Tp-2015-218570 [Internet]. 2015;(March):1-220.

22. Anderson. Summary of Water Processing Trade Studies and Analysis of JSC Integrated Water Recovery System (IWRS) [Internet]. 2004. Available from: http://www.marsjournal.org/contents/2006/0005/files/Anderson2004.pdf

23. Fukui, T., Shiomi, N., \& Doi, Y. Expression and Characterization of (R)-Specific Enoyl Coenzyme A Hydratase Involved in Polyhydroxyalkanoate Biosynthesis by Aeromonas caviae. Journal of Bacteriology. 1998;180(3): 667-73.

24. McCool, G. J., \& Cannon, M. C. Polyhydroxyalkanoate Inclusion Body-Associated Proteins and Coding Region in Bacillus megaterium. Journal of Bacteriology. 1999;181(2), 585-592.

25. Blenner M. Synthetic Biology for Recycling Human Waste into Nutraceuticals and Materials: Closing the Loop for Long-Term Space Travel [Internet]. 2015. Available from: https://www.nasa.gov/feature/synthetic-biology-forrecycling-human-waste-into-nutraceuticals-and-materials-closing-the 


\section{pET29b(+)-phaCJ}

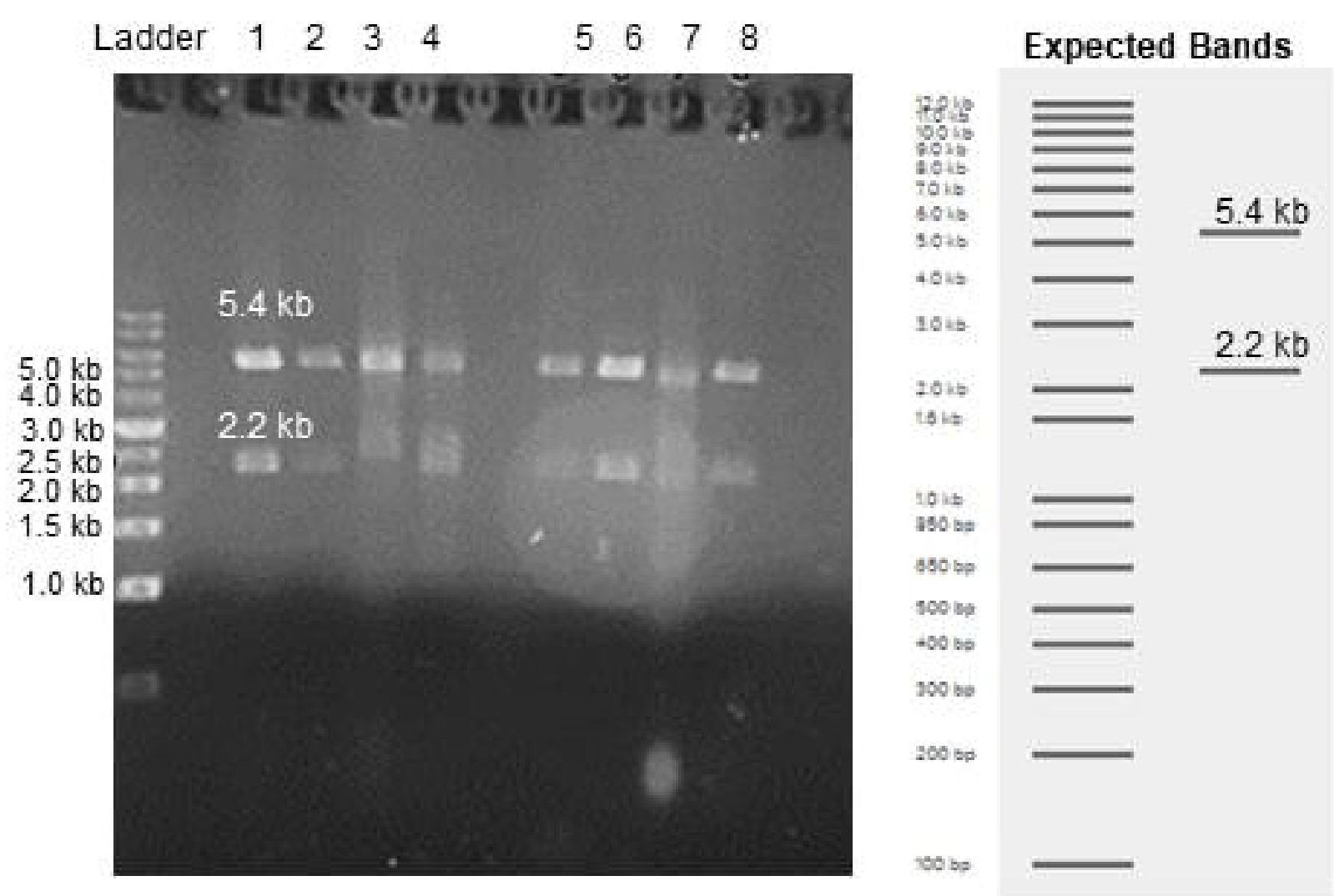




\section{pET29b(+) -phaCBA}

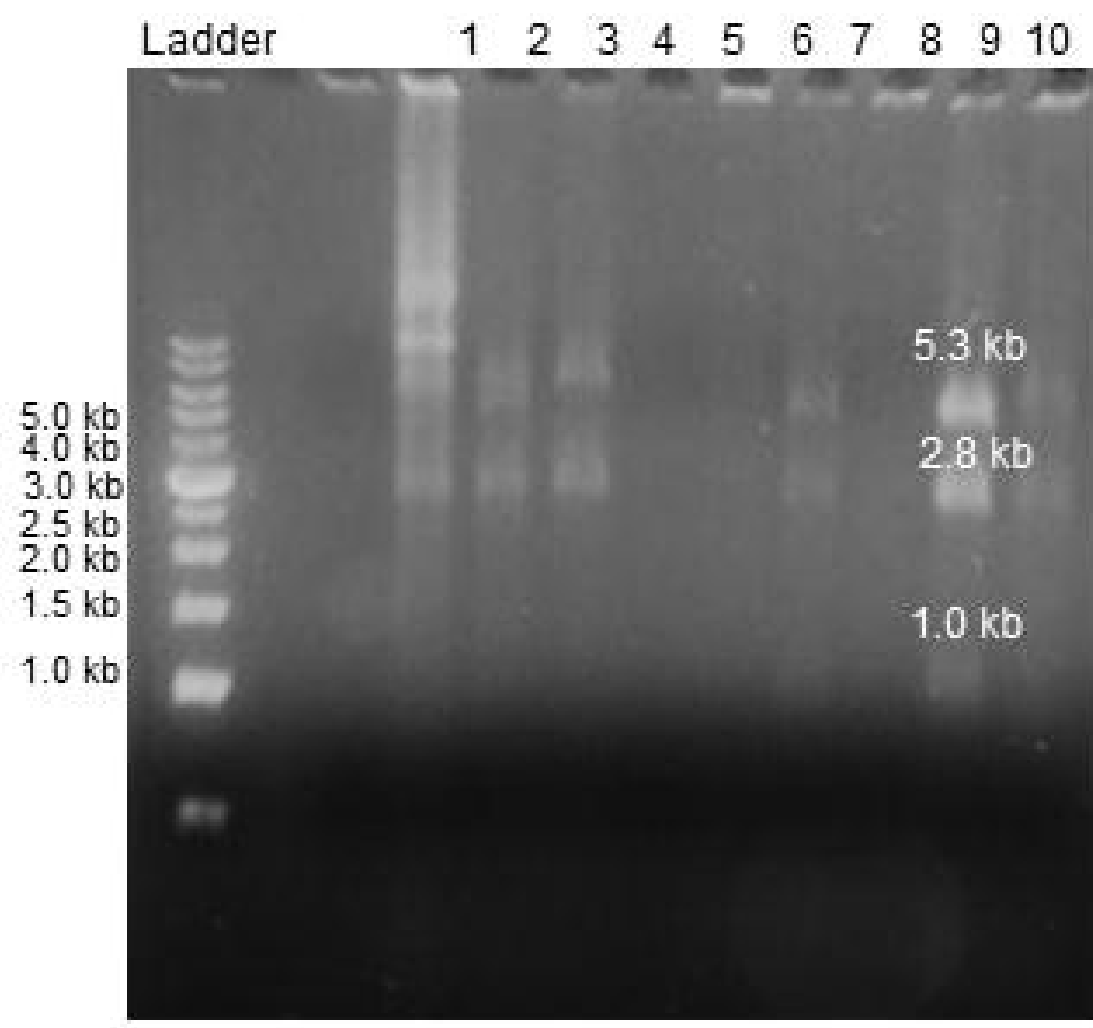

Expected Bands

soins

aove

$50 \mathrm{kt}$

190

dout

$20 \mathrm{~km}$

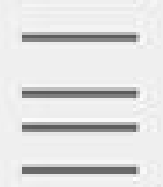

$5.3 \mathrm{~kb}$

$\underline{ }$

$2.8 \mathrm{~kb}$

20,

isis

$12 \mathrm{in}$

$10 \%$

ion of

$706 \mathrm{ba}$

400 ba

200 bp

405 be

300 bo

200 bs

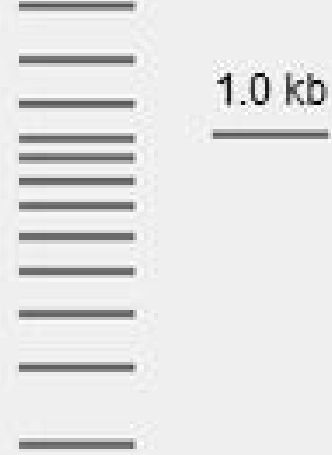

$16 \mathrm{bp}$ 


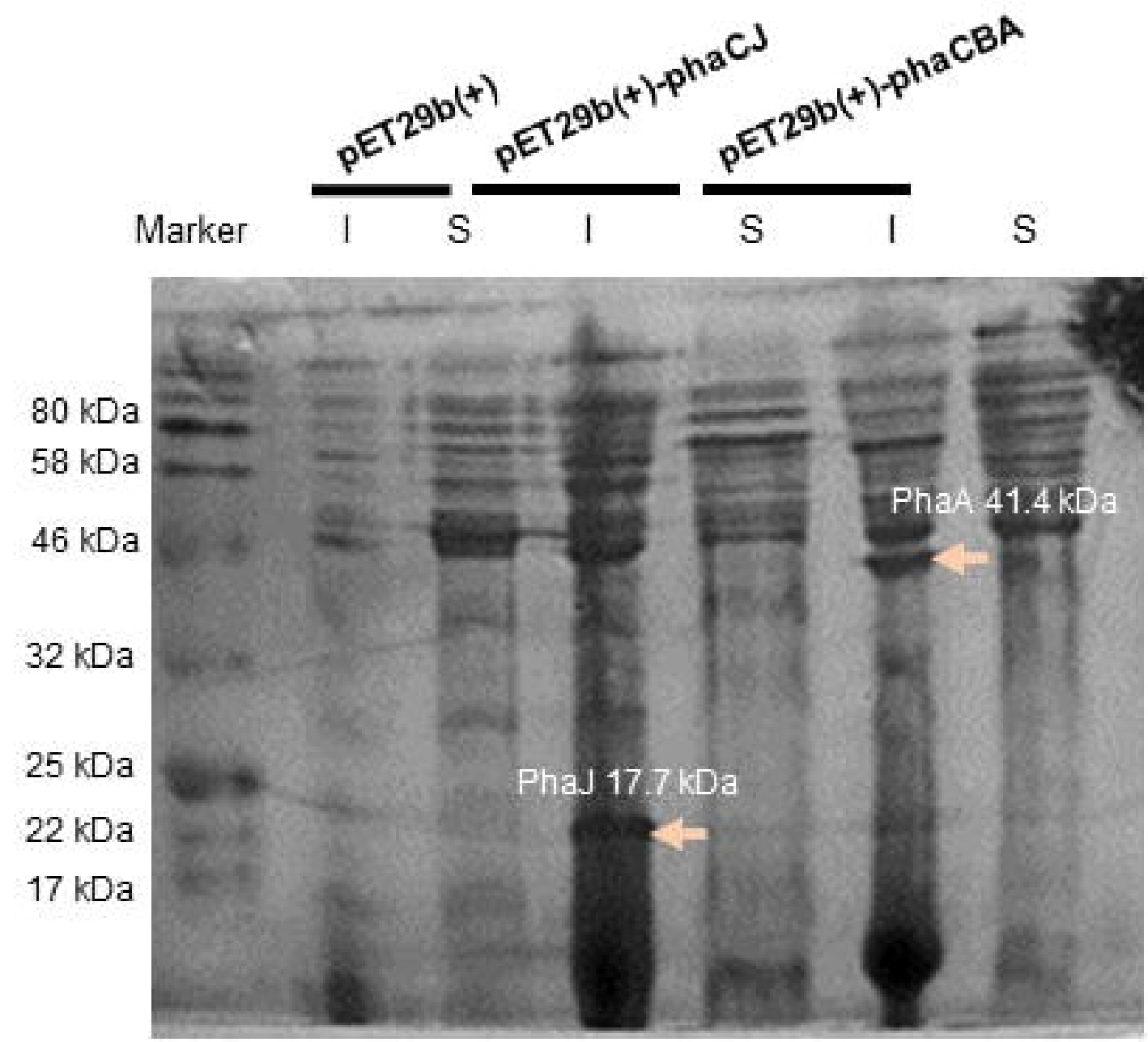




\section{pET29b(+) pET29b(+)-phaCJ}

FSPS

FSPS

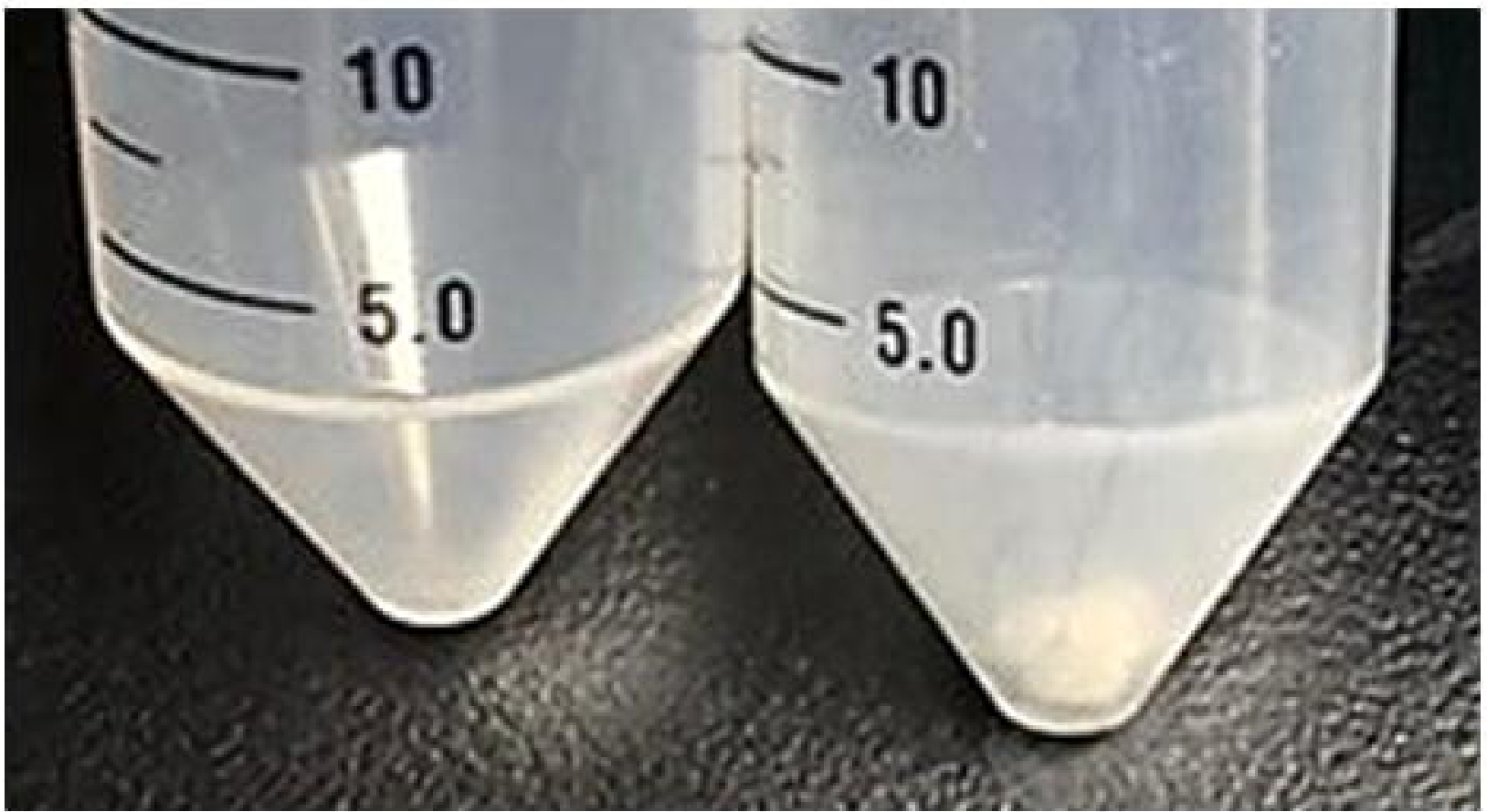




\section{pET29b(+) pET29b(+)-phaCBA FSPS FSPS}

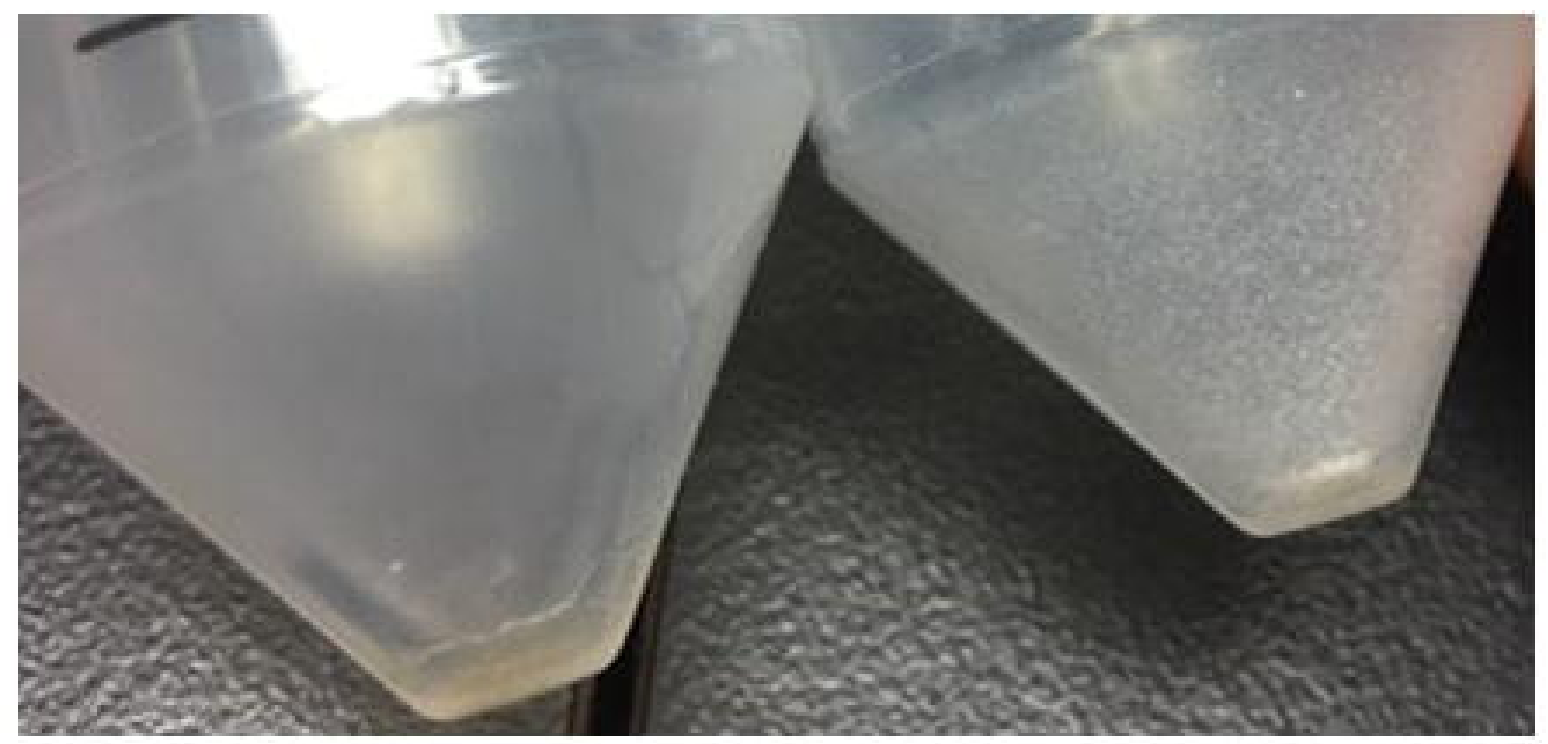




\section{\begin{tabular}{|c|}
\hline Chromatogram \\
\hline
\end{tabular}}

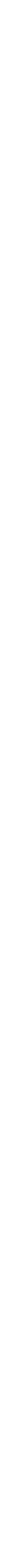

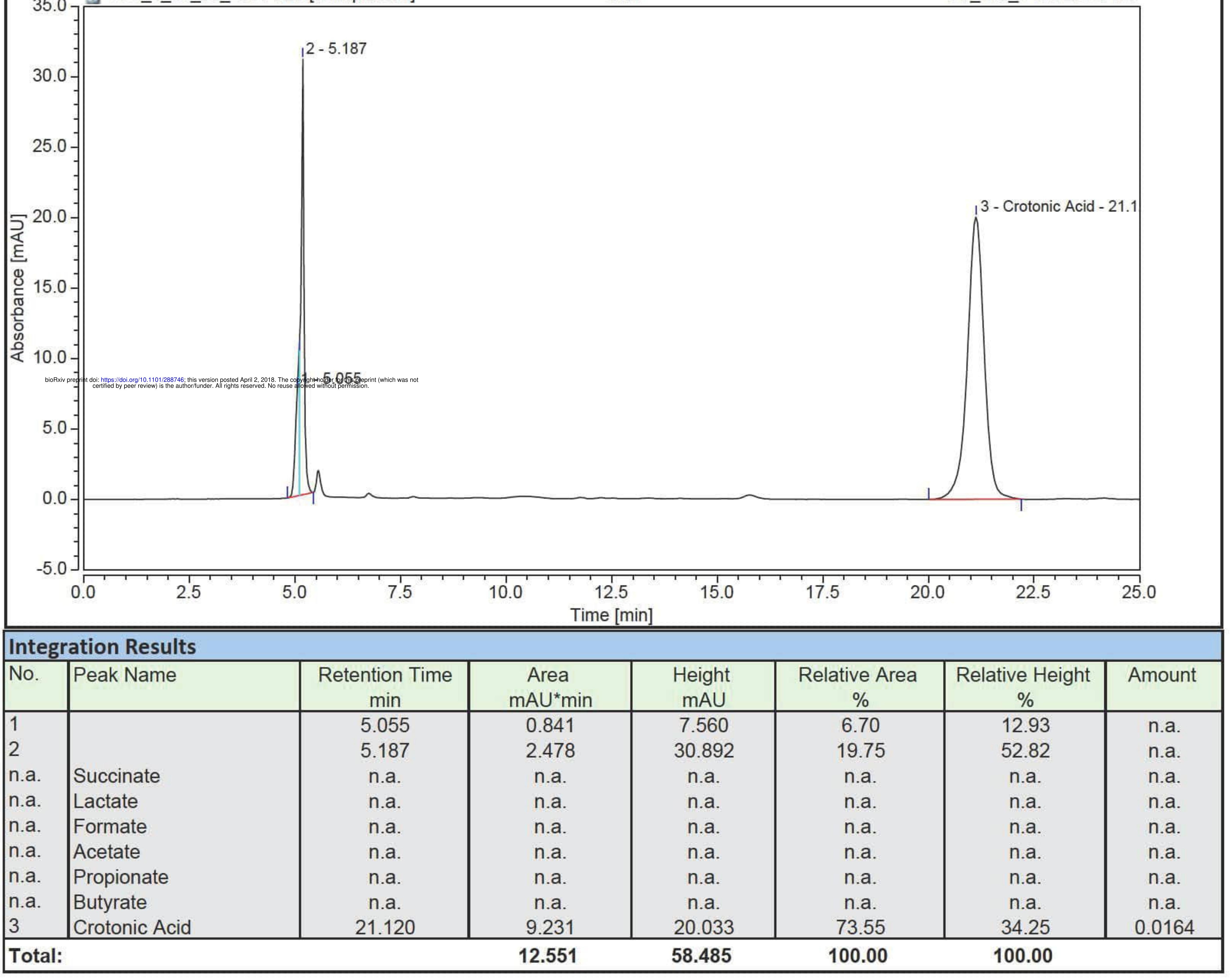




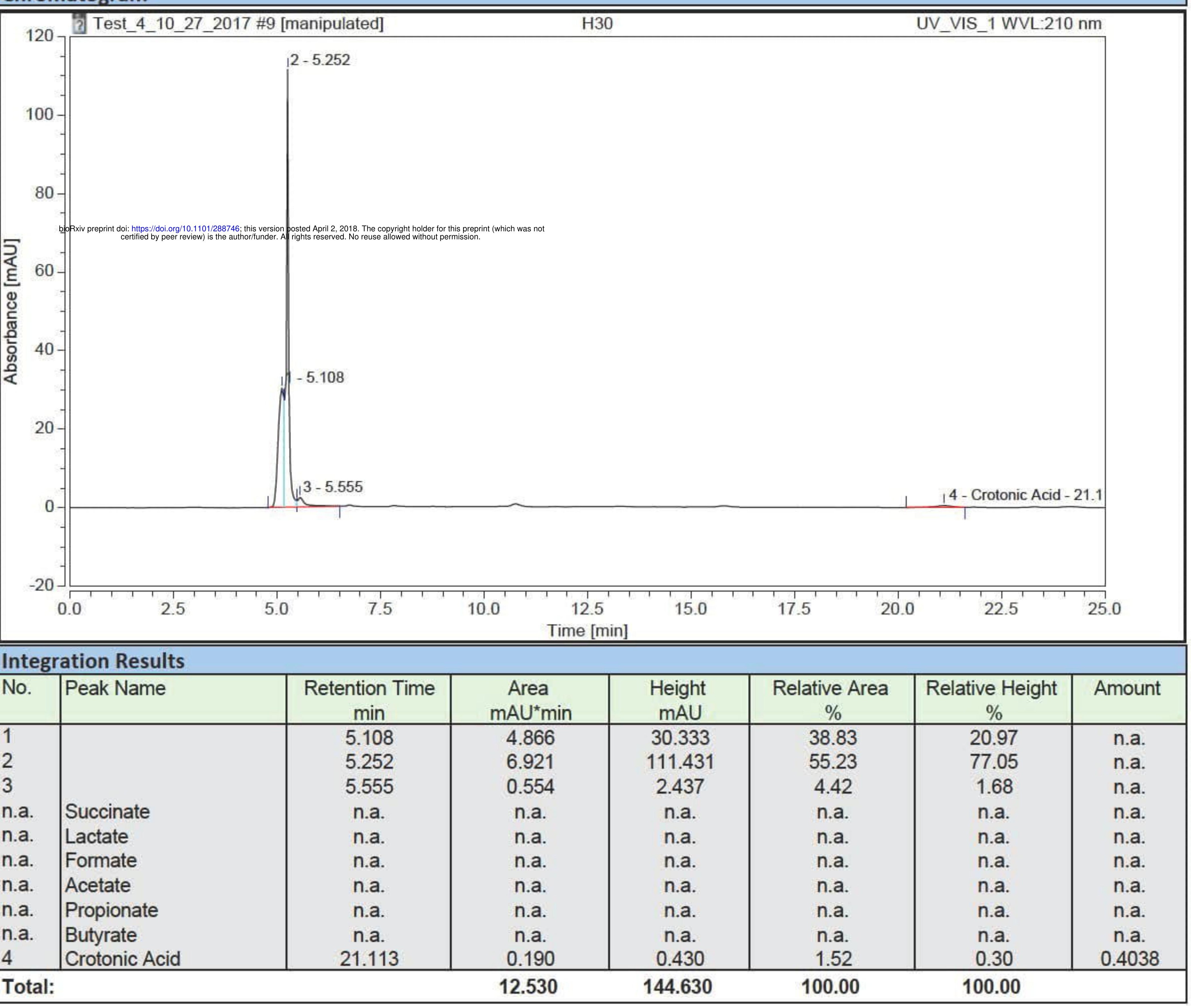

Integration Results 
L U DD

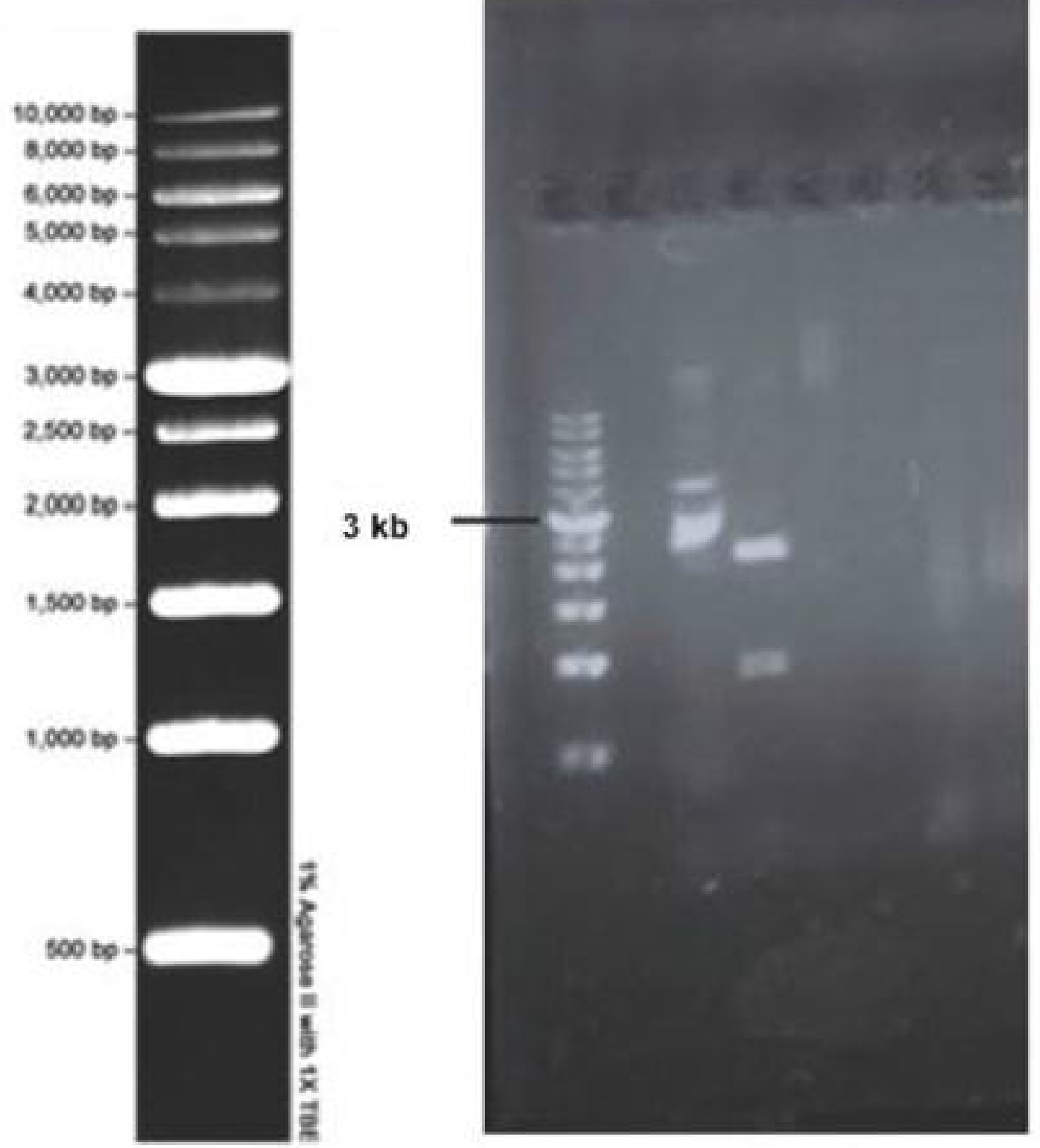

L

DD

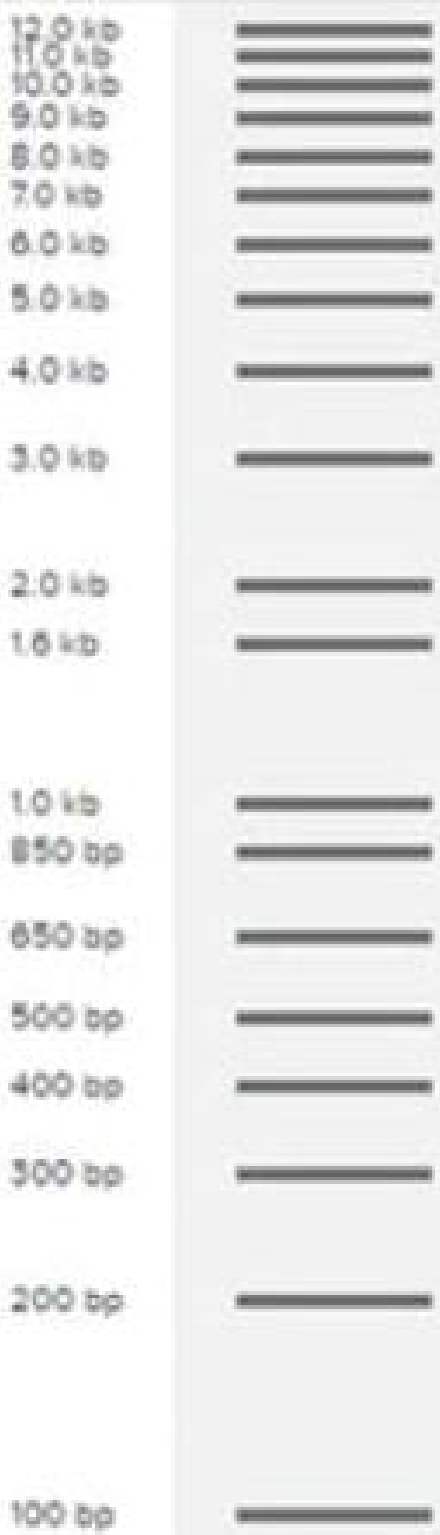

$2.0 \mathrm{~kb}$

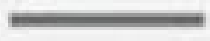

889 bp 


\section{VFA composition after 3 days of fermentation at $22^{\circ} \mathrm{C}$ and $37^{\circ} \mathrm{C}$}

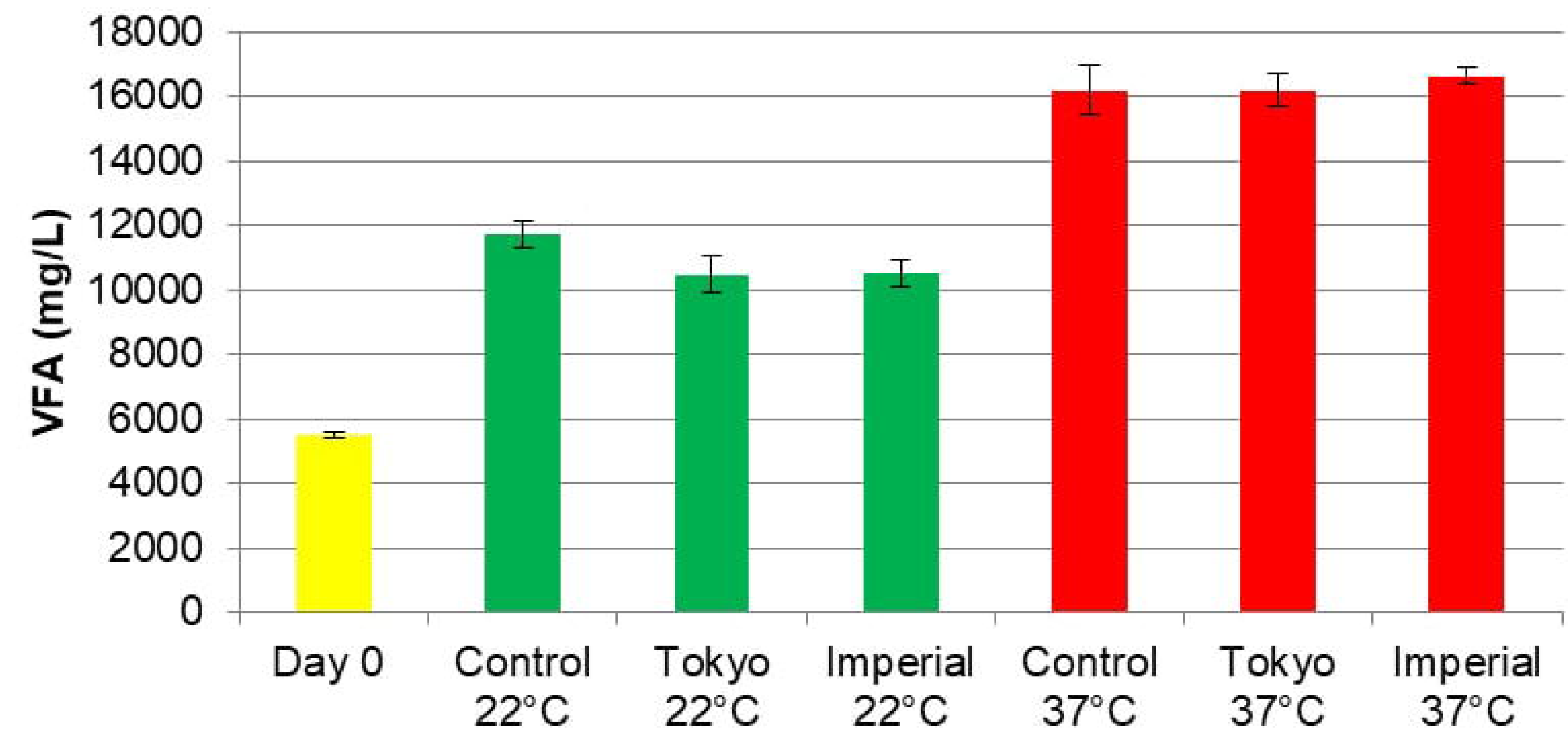


VFA composition after 3 and 5 days of fermentation at $22^{\circ} \mathrm{C}$ and $37^{\circ} \mathrm{C}$

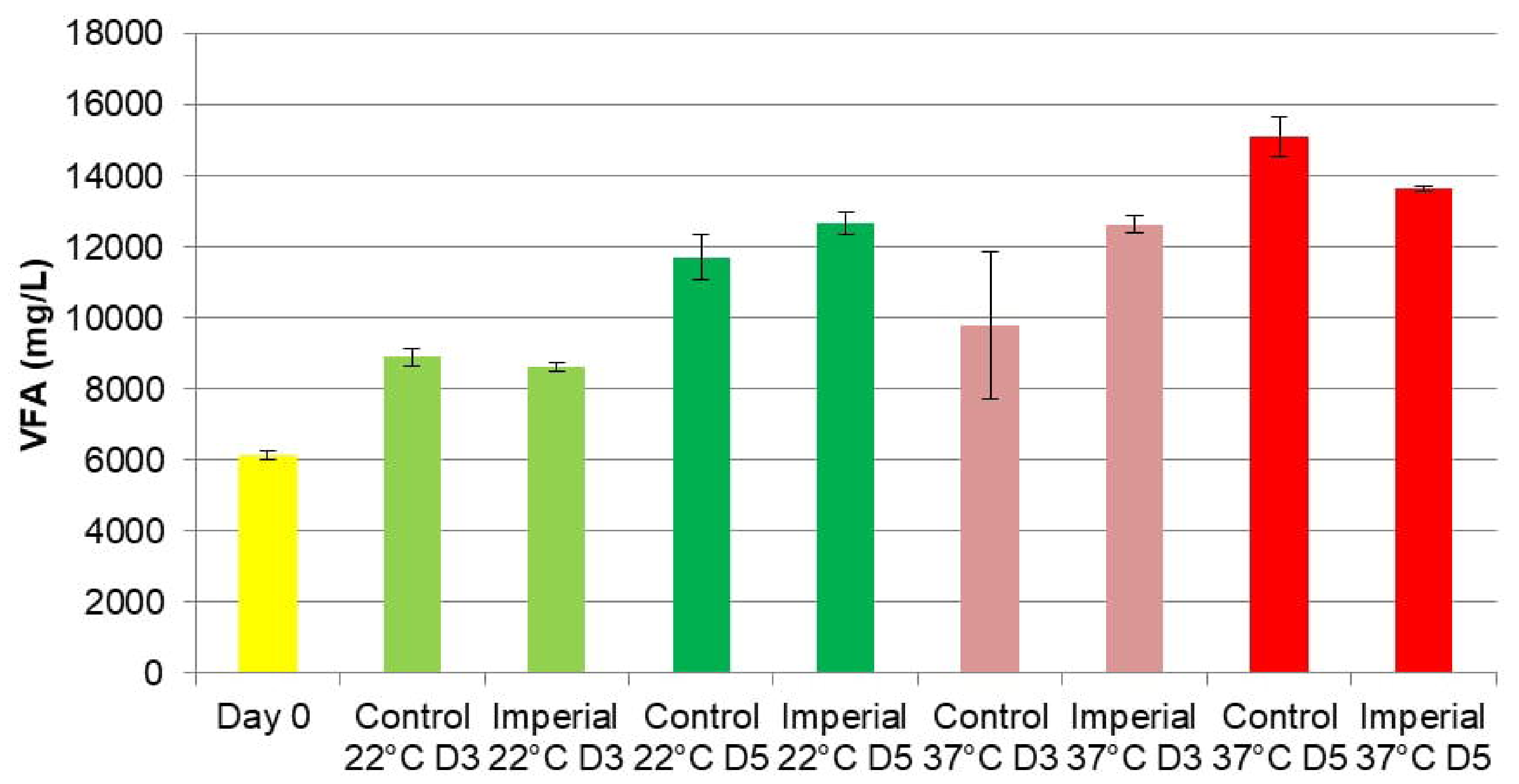




\section{Extraction Methods}
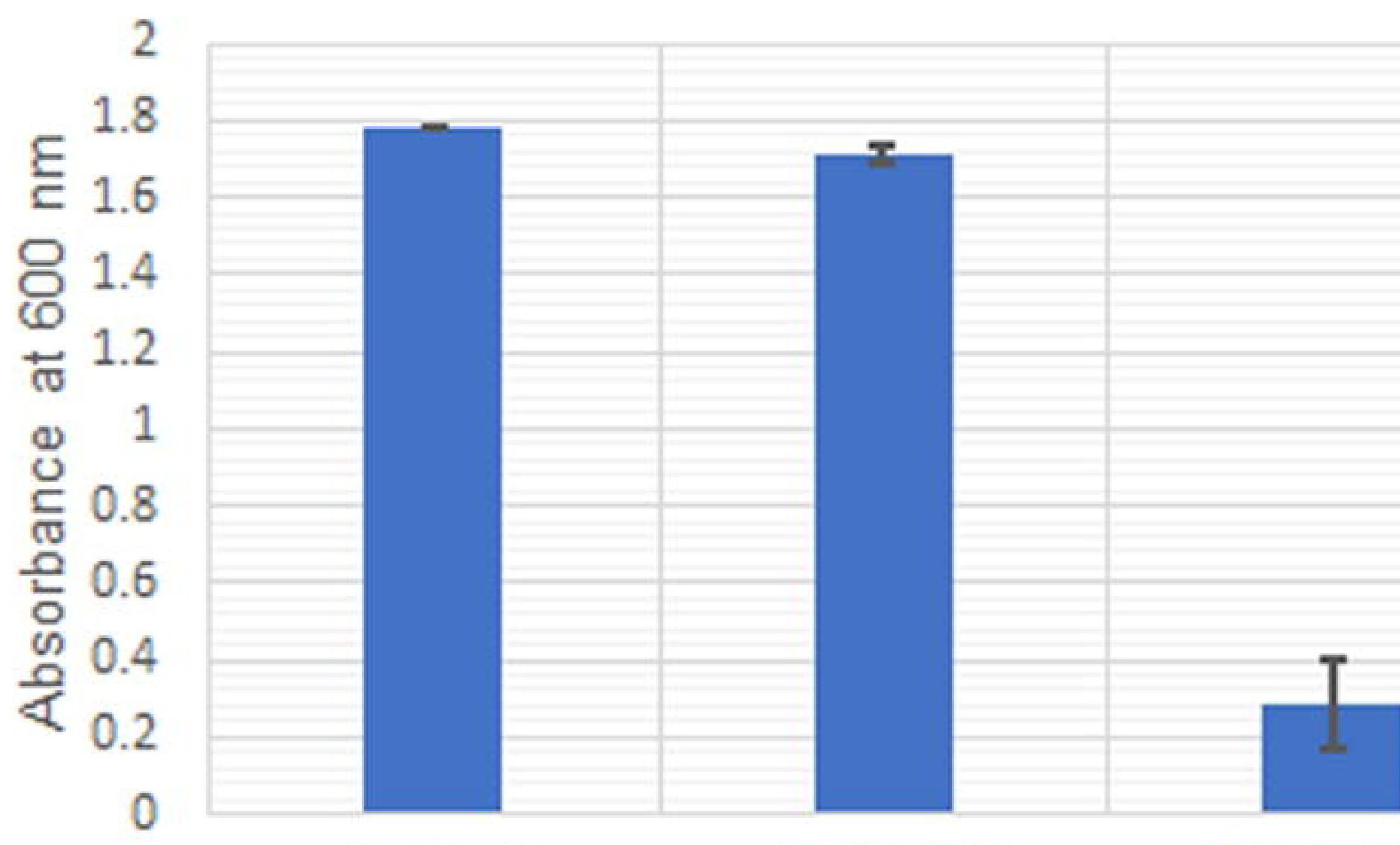

Original

1000 RPM

3750 RPM

$10 \mathrm{mM} \mathrm{CaCl} 2+$ 3750 RPM

Conditions Tested 
FECES \& WATER

CENTRIFUGAL
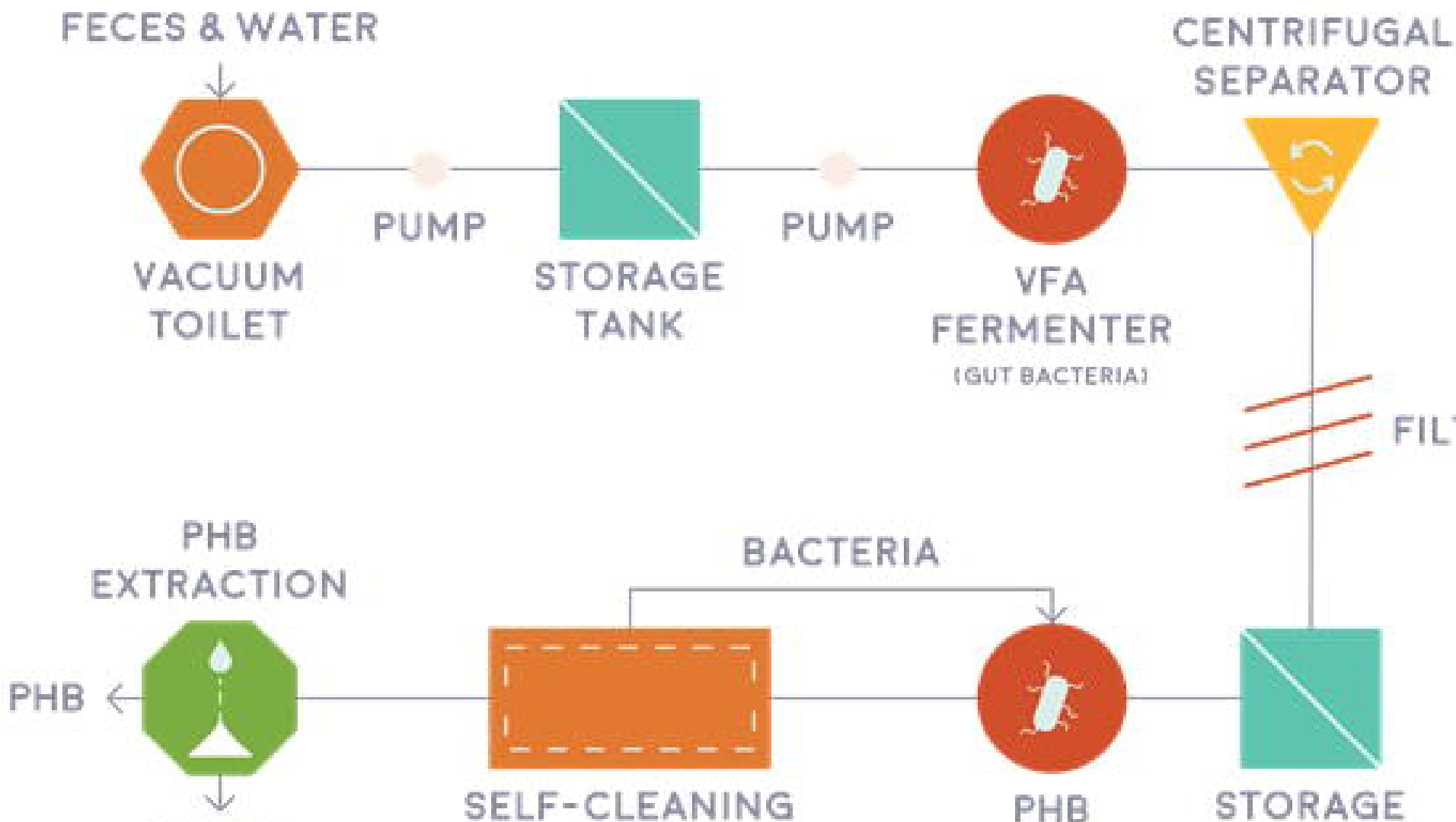

WATER

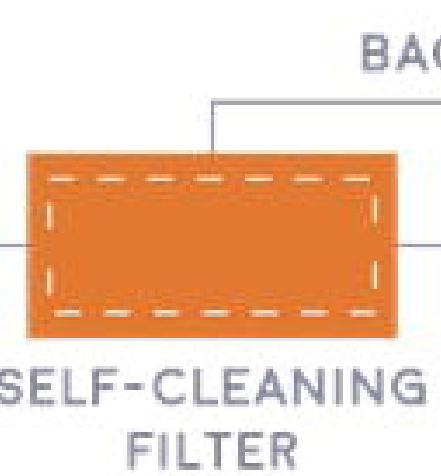

BACTERIA

FERMENTER

(GUT BACTERIA)

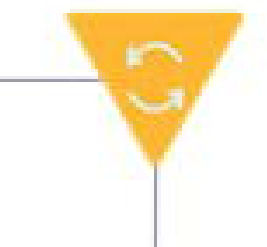

ON 
WATER PURIFICATION

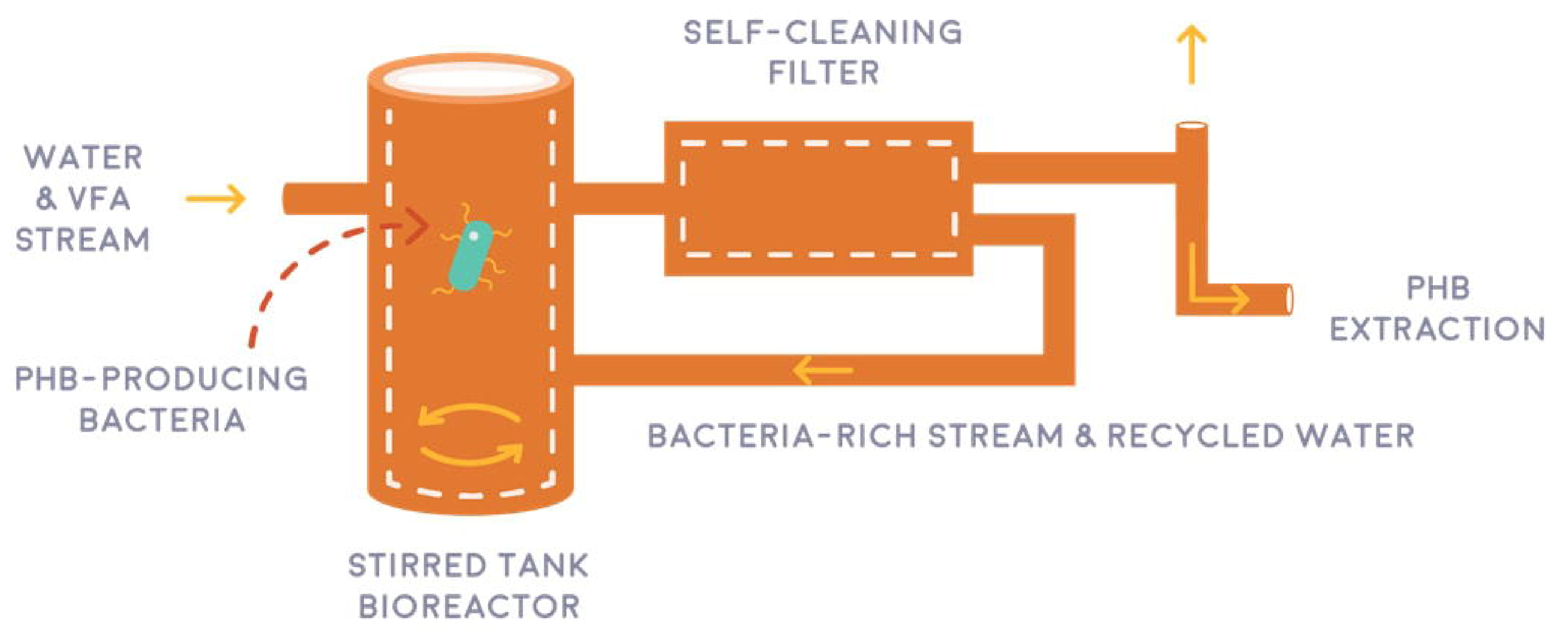




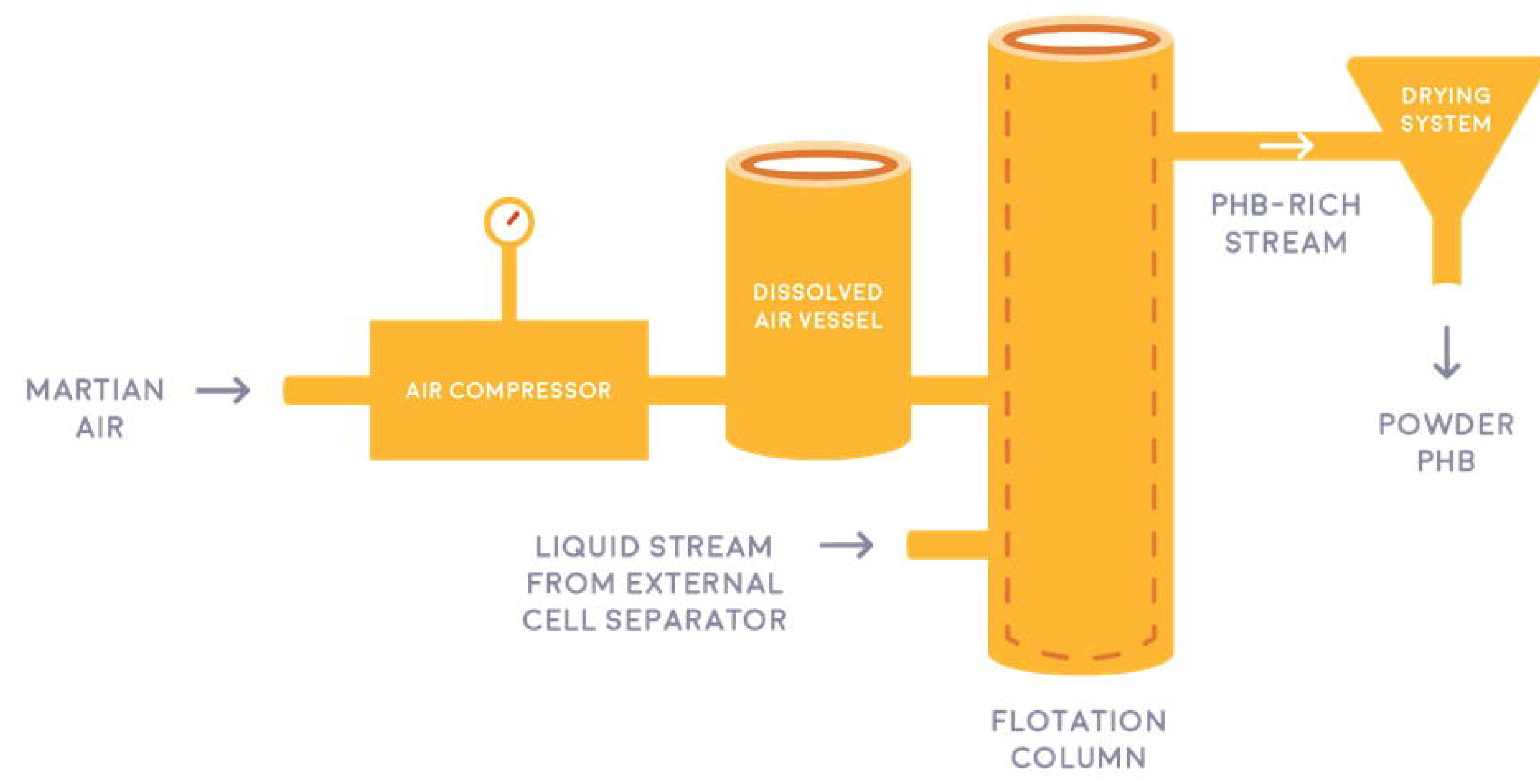


0.25

0.17251

0,2

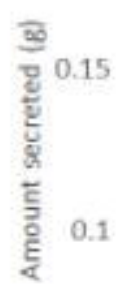

0.12417
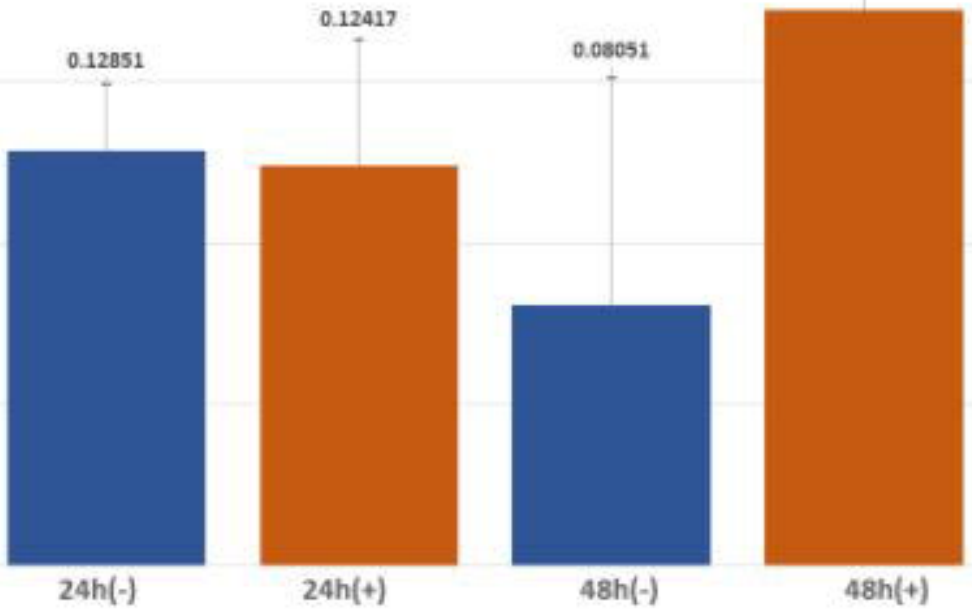

$48 h(+)$ 